\title{
Digital Predistortion of Phased Array Transmitter With Shared Feedback and Far-Field Calibration
}

\author{
Nuutti Tervo, Student Member, IEEE, Bilal Khan, Olli Kursu, Janne P. Aikio, Markku Jokinen, \\ Marko E. Leinonen, Student Member, IEEE, Markku Juntti, Fellow, IEEE, \\ Timo Rahkonen, Senior Member, IEEE, and Aarno Pärssinen Senior Member, IEEE
}

\begin{abstract}
Digital predistortion (DPD) of a phased array requires that multiple transmit paths must be measured by a feedback (FB) receiver $(\mathrm{Rx})$. In this paper, we propose a FB concept for DPD in a time-division-duplex (TDD) phased arrays. We use a single FB line to collect the waveform samples from the parallel transmit paths to the FB Rx. The TDD switches are used to enable and disable individual transmit paths. The feedback is calibrated by comparing the FB outputs from individual PAs to over-the-air (OTA) measurement reference performed with a frequency modulated continuous wave (FMCW) signal. The individual PA measurements are post-equalized before the DPD training to model the far-field signal. Three alternative strategies are considered for training the DPD through the calibrated FB line and compared with the OTA DPD. The performance is verified by OTA measurements of a $28 \mathbf{~ G H z}$ phased array transmitter and with fifth generation New Radio waveform in terms of total radiated (TR) adjacent channel power ratio (ACPR), cumulative absolute ACP (CACP), and main lobe error vector magnitude (EVM). The best EVM and ACPR performance is achieved by the strategy where the individual PA responses are treated independently. The methods were comparable to the OTA DPD performance, achieving all $<37$ dB TRACPR, $-29 \mathbf{~ d B m} / \mathbf{M H z} \mathbf{C A C P}$, and $\leq 7 \%$ EVM.
\end{abstract}

Index Terms-Antenna array, adjacent channel power ratio (ACPR), beamforming, digital predistortion (DPD), feedback receiver, gallium nitride (GaN), linearization, over-the-air (OTA)calibration, fifth generation (5G) New Radio (NR).

\section{INTRODUCTION}

$\mathbf{F}$ IFTH generation (5G) [1] and upcoming sixth generation (6G) [2] systems utilize high carrier frequencies for wireless communications. Highly directive antennas and analog beamforming are being used to implement directive communications with limited digital signal processing resources. High data-rate targets require either extremely high bandwidth or high spectral efficiency. In sub $6 \mathrm{GHz}$ frequencies (FR1), 256-quadrature amplitude modulation (QAM) and even higher modulations are used, while in millimeter-wave $(\mathrm{mmW})$ frequencies (FR2 bands) Third Generation Partnership Project (3GPP) has specified modulations up to 64-QAM for 5G New Radio (NR).

This paper is an extended version from the IEEE MTT-S International Symposium (IMS2020), virtual event, August 4-6, 2020. Manuscript received on July 8, 2020; revised September 15, 2020; accepted October 10, 2020.

The authors are with Department of Information Technology and Electrical Engineering (ITEE), University of Oulu, 90570 Oulu, e-mail: nuutti.tervo@oulu.fi. The research leading to this paper have received funding from Business Finland 5G-VIIMA project, Academy of Finland 6Genesis Flagship (grant no. 318927) and Infotech Oulu Doctoral Programme.
High-order modulations and orthogonal frequency division multiplexing (OFDM) waveform set stringent linearity requirements for the phased array transmitter (Tx) in order to achieve low error vector magnitude (EVM). On the contrary, the adjacent channel power ratio (ACPR) specifications for 5G FR2 Tx are relatively loose [1], [3]. One way to improve the Tx linearity is to use digital predistortion (DPD). However, due to the analog beamforming architectures, multiple parallel nonlinear elements have to be linearized by a single DPD. Many different methods for phased array DPD are proposed in the literature and most of them aim for minimizing the radiated distortion. For achieving a low EVM level, the main target of the DPD is in the beamforming direction. Directive DPD has been proposed for example in [4]-[9].

\section{A. State-of-the-art Array Feedback Rx Architectures}

DPD requires a feedback $(\mathrm{FB})$ receiver $(\mathrm{Rx})$ to measure the nonlinear output of the device under test (DUT). For an array, the nonlinear output consists of multiple parallel elements. Hence, the FB strategy plays a crucial role in the array DPD concept as the measurements through the FB are eventually used to train the DPD. Several array FB architectures have been proposed in the literature. In general, they can be divided into conductive (e.g. [4]-[7], [9]-[11]), and over-the-air (OTA) (e.g. [3], [8], [12]-[17]) FB methods. The OTA methods provide low-complexity ways to measure the radiated response. However, far-field distance of large arrays and potential multipath propagation makes the approach challenging to apply in real systems, where very compact array designs [18]-[20] are used. Also, arranging a proper reference antenna setup is not straightforward in the field, for example if the base stations (BSs) are installed in very different places. Furthermore, the far-field reference antenna may interfere with the beam transmitted by the array, and it is also potentially affected by the interference from the other systems.

Coupling antennas in near-field are used in [13], but those can effectively only collect paths connected to the adjacent antenna elements without significant attenuation and require breaking the uniform array geometry. The conductive FB architectures contain a single power amplifier (PA) FB [10], switchable FB [4], [5], [7] and combined anti-beamforming or phased FB [6], [11]. If the paths have different nonlinear characteristics, single PA FB is not enough to build a decent DPD object. On the contrary, the combined phased FB architectures have high radio frequency $(\mathrm{RF})$ complexity due to 


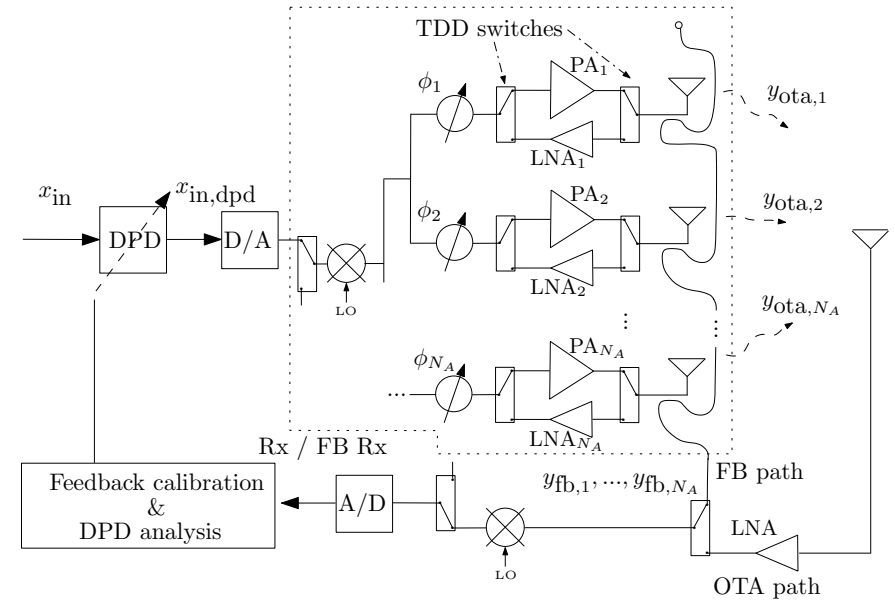

Fig. 1. Phased array Tx with DPD using the proposed combined FB architecture and OTA calibration. The blocks inside the dotted box are implemented on the demonstration PCB [21].

the additional analog beamforming performed in the FB path. Switchable FB architectures aim to collect each PA at different time instants. All state-of-the-art switchable FB architectures use additional switches in the feedback to connect the FB Rx to the PA outputs one-by-one. In such a time sharing scheme, it is also challenging to observe the phase of the output in a time-domain measurement. Further details of the feedback approaches are presented in Section III.

\section{B. Proposed Feedback Concept and Contributions}

The applicable FB strategy depends on the used transceiver (TRx) array architecture. In the time-division-duplex (TDD) TRx architecture used in this paper, the Tx and Rx paths share the same passive power combining network and phase shifters. In such an architecture, the TDD switches enable to switch the individual elements, and hence the active PAs one-by-one. This can be also utilized when designing the FB approach. The FB architecture presented in this paper contains a FB line shared by multiple transmit paths and was originally presented in [21]. The basic concept is depicted in Fig. 1. The dotted line surrounds the content of the implemented printed circuit board (PCB). Compared to the existing FB architectures, this offers an alternative way of training the DPD without using additional switches or beamforming in the FB path. The amplitude and phase of the FB line from each coupling point to the FB output is post-equalized by comparing the FB measurements to the OTA calibration measurements. After a single FB calibration, the reference antenna is not required for DPD training.

In [21], the same modulated signal is used for both feedback calibration and DPD training. This compromises the calibration performance by focusing mostly on the main signal band (i.e. EVM) due to the low calibration signal level in the adjacent channels. For linearization, the signal content in the adjacent channel is of particular importance when training the DPD with oversampled signals. In this paper, we use frequency modulated continuous wave (FMCW) (i.e. a frequency sweep), signal to characterize the feedback fre- quency responses over the used FB bandwidth. This extents the calibration to cover also the adjacent bands. Furthermore, as an extension to [21] we analyze and review different feedback approaches, present three different DPD strategies that use the measurements through the proposed FB, show detailed analysis on the feedback calibration, and present the results of spatially distributed ACPR, total radiated ACPR, and the main lobe EVM, together with azimuth domain beamsteering.

The rest of the paper is organized as follows. Section II introduces the array nonlinearity and the system level figures of merit (FoMs) used to characterize and specify it. Alternative feedback solutions for training the DPD are discussed in Section III. The used feedback architecture, demonstration platform, and feedback calibration measurements are presented in Section IV. Three alternative DPD strategies trained through the feedback are presented in Section V, followed by OTA measurements and validation of the used linearization technique in Section VI. Section VII concludes the paper.

\section{Radiated NONLINEAR Distortion FROM PHASED ARRAYS}

\section{A. PA Nonlinearity versus Array Nonlinearity}

In single antenna systems, the nonlinearity charaterized at the PA output is usually the same as the radiated nonlinearity divided by the antenna efficiency. This is due to the fact that the antenna itself fundamentally only directs the signal, but does not add any nonlinear content to it. Hence, the waveform samples in the frequency domain, radiated to each spatial direction can be simply calculated as [22]

$$
Y_{\mathrm{SE}}(f, \phi, \theta)=\eta_{\mathrm{A}}(f) Y_{\mathrm{PA}}(f) F_{\mathrm{SE}}(f, \phi, \theta),
$$

where $f$ denotes the frequency, $\eta_{\mathrm{A}}(f)$ denotes the total antenna efficiency, $Y_{\mathrm{PA}}(f)$ denotes the PA output signal (antenna input), and $F_{\mathrm{SE}}(f, \phi, \theta)$ denotes the antenna pattern at azimuth angle $\phi$ and elevation angle $\theta$.

In an antenna array, the nonlinear distortion can also be beamformed and it may even have different beam pattern compared to the linear part of the signal as discussed in [4], [11]. This is due to the fact that the nonlinear signal radiated by each antenna is different due to the varying PA characteristics [3], [11], beamforming [4], active load pulling from the neighbouring elements due to the finite antenna isolation [23], [24], and antenna impedance variations [25]. The frequency domain waveform samples radiated by the antenna array to each spatial direction can be characterized as

$$
Y_{\mathrm{A}}(f, \phi, \theta)=\sum_{i=1}^{N_{\mathrm{A}}} \eta_{i}(f) Y_{i}(f) F_{i}(f, \phi, \theta) e^{j \vec{k}^{T} \vec{r}_{i}},
$$

where $N_{\mathrm{A}}$ is the number of antenna elements, $\eta_{i}$ denotes the total antenna efficiency of $i$ th antenna branch, $F_{i}(f, \phi, \theta)$ is the antenna pattern, $\vec{k}=\frac{2 \pi}{\lambda}[\sin (\theta) \cos (\phi), \sin (\theta) \sin (\phi), \cos (\theta)]^{T}$ is the three-dimensional (3D) wave vector, and $\vec{r}_{i}=$ $\left[r_{x}, r_{y}, r_{z}\right]^{T}$ are the element coordinates in $3 \mathrm{D}$ space. Notation ()$^{T}$ stands for the transpose and $j$ is the imaginary unit. $Y_{i}(f)$ denote the signal at each antenna which consists the waveform 
of the $i$ th branch and the coupled signals from other branches as

$$
Y_{i}(f)=Y_{\mathrm{PA}, i}(f)+\sum_{\substack{l=1, j \neq i}}^{N_{\mathrm{A}}} Y_{l}(f) S_{i l}(f),
$$

where $S_{i l}(f)$ is the scattering parameter (S-parameter) from antenna port $l$ to antenna port $i$.

\section{B. Phased Array Figures of Merit of Nonlinearity}

In the system level, the nonlinearity is conventionally characterized in terms of EVM and ACPR. EVM is a FoM that describes the modulation error of the signal against the reference constellation and is affected by all RF nonidealities, e.g., thermal noise, phase noise, in-phase quadrature-phase (IQ) imbalance, etc. [26], [27]. Hence, EVM is limited by the dominant RF nonideality of the system which is not always the PA nonlinearity, especially when the PAs are linearized by DPD. For antenna arrays, the EVM is characterized in the desired steering angle [1]. 3GPP 5G NR FR2 (i.e. mmW) Tx EVM specifications are given in Table I.

TABLE I

5G NR FR2 TX EVM SPECIFICATIONS. *SPECIFICATION FOR 256-QAM IS TAKEN FROM FR 1 .

\begin{tabular}{|c|c|c|c|c|}
\hline Modulation & QPSK & 16-QAM & 64-QAM & 256-QAM* \\
\hline Tx EVM (\%) & 17.5 & 12.5 & 8.0 & 3.5 \\
\hline
\end{tabular}

ACPR is interference for the systems and users operating in the adjacent channels. For 5G FR2 Tx, 3GPP specifies the ACPR as the total radiated power (TRP). In general, this means that the system does not care about the spatial direction of the adjacent channel power (ACP), but integrates the signal power over the angular domains. For a discrete set of measured directions, this can be approximated as

$$
P_{\mathrm{TRP}} \approx \frac{\pi}{2 N_{a z} N_{\mathrm{el}}} \sum_{n=0}^{N_{a z}-1} \sum_{m=0}^{N_{\mathrm{el}}-1} \operatorname{EIRP}\left(\phi_{n}, \theta_{m}\right) \sin \theta_{n}
$$

where $\operatorname{EIRP}\left(\phi_{n}, \theta_{m}\right)$ denotes the effective isotropic radiated power observed at azimuth angle $\phi_{n}$ and elevation angle $\theta_{m}$. The EIRP is calculated as a sum over both linear polarizations. $N_{\mathrm{az}}$ and $N_{\mathrm{el}}$ are the number of elevation and azimuth angles, respectively. The total radiated adjacent channel power ratio (TRACPR) in linear scale can be calculated as

$$
\begin{aligned}
& \text { TRACPR }=\frac{\text { TRACP }}{\text { TRCP }} \\
& =\frac{\frac{\pi}{2 N_{a z} N_{\mathrm{el}}} \sum_{n=0}^{N_{a z}-1} \sum_{m=0}^{N_{\mathrm{el}}-1} P_{\mathrm{CH}}\left(\phi_{n}, \theta_{m}\right) \sin \theta_{n}}{\frac{\pi}{2 N_{a z} N_{\mathrm{el}}} \sum_{n=0}^{N_{a z}-1} \sum_{m=0}^{N_{\mathrm{el}}-1} P_{\mathrm{ACP}}\left(\phi_{n}, \theta_{m}\right) \sin \theta_{n}} \\
& =\frac{\sum_{n=0}^{N_{a z}-1} \sum_{m=0}^{N_{\mathrm{el}}-1} P_{\mathrm{CH}}\left(\phi_{n}, \theta_{m}\right) \sin \theta_{n}}{\sum_{n=0}^{N_{a z}-1} \sum_{m=0}^{N_{\mathrm{el}}-1} P_{\mathrm{ACP}}\left(\phi_{n}, \theta_{m}\right) \sin \theta_{n}},
\end{aligned}
$$

where TRCP and TRACP denotes the total radiated channel power and adjacent channel power, respectively.
In general, if we assume that the antenna efficiency is approximately the same for the adjacent channels and main channel, the TRACPR can also be calculated as

$$
\operatorname{TRACPR}=\frac{\sum_{i=1}^{N_{\mathrm{PA}}} P_{\mathrm{ch}, i}}{\sum_{i=1}^{N_{\mathrm{PA}}} P_{\mathrm{ACP}, i}}
$$

where $P_{\mathrm{ch}, i}$ denotes the root-mean-square (RMS) channel power of branch $i$ and $P_{\mathrm{ACP}, i}$ is the RMS adjacent channel power of branch $i$. Often the ACP is calculated as the maximum of the higher and lower adjacent channel powers, i.e. $P_{\mathrm{ACP}}=\max \left(P_{\mathrm{ACP}_{\mathrm{L}}}, P_{\mathrm{ACP}_{\mathrm{H}}}\right)$.

It is important to note that the TRP measurement itself is very sensitive for the angular grid, noise and other measurement imperfections [28]. This is why the 3GPP test specifications [29] relax the ACPR even further compared to the system specifications [1], depending on the measurement method [3]. In other words, the uncertainty of the OTA measurement is given for the manufacturers' benefit. The Tx ACPR specifications are given in Table II for center frequencies $24.25-33.4 \mathrm{GHz}$. In addition to the relative ACPR, 3GPP specifies also the cumulative ACPR (CACPR) in terms of absolute power (per frequency unit). CACPR denotes the power integrated over the adjacent channels. Note that for a FR2 base station (BS) transmitter, 3GPP does not specify the limit for the absolute main channel TRP. When directly calculated from the CACPR and TRACPR limits, the Tx OTA TRP in the case of $-28 \mathrm{~dB}$ ACPR would be only $31 \mathrm{dBm}$. In other words, if the system can improve the linearity further beyond the given ACPR specifications, it can provide significantly more output power. This motivates the DPD especially for higher power systems aiming at longer link ranges and larger cell sizes. However, the EIRP is often specified by other local regulations and authorities [30].

TABLE II

5G NR FR2 TX ACPR SPECIFICATIONS GIVEN IN TRP.

\begin{tabular}{|c|c|c|}
\hline Parameter & Tx spec. [1] & Tx meas spec. [29] \\
\hline ACPR $(\mathrm{dB})$ & -28.0 & -25.7 \\
\hline CACPR $(\mathrm{dBm} / \mathrm{MHz})$ & -20.0 & -17.3 \\
\hline CACPR $(\mathrm{dBm} / 200 \mathrm{MHz})$ & 3.0 & 5.7 \\
\hline
\end{tabular}

\section{FEedBACK Strategies for Phased ARray DPD TRAINING}

The used FB Rx architecture sets the framework for the array linearization strategy. Different feedback architectures have major differences in their implementation complexity. On the other hand, they also enable the observation of different aspects that may be important for the system. For example, if the system is able to measure each PA output individually, it is possible to estimate the total radiated distortion based on (6) without the actual beam measurement. The feedback architectures used in the literature can be divided into conductive and OTA methods. In the following subsections, we review and discuss the different feedback approaches. 


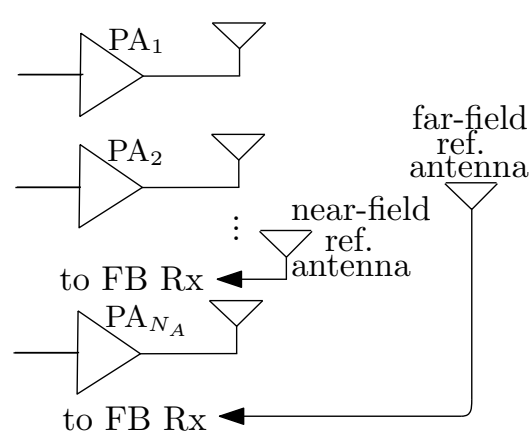

(a)

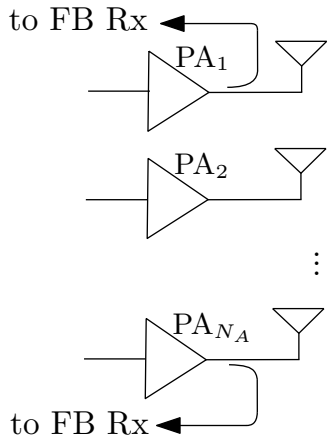

(b)

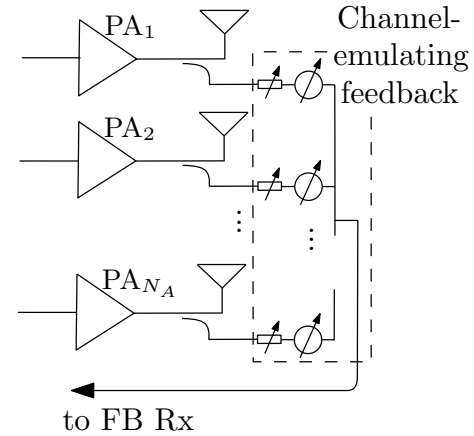

(c)

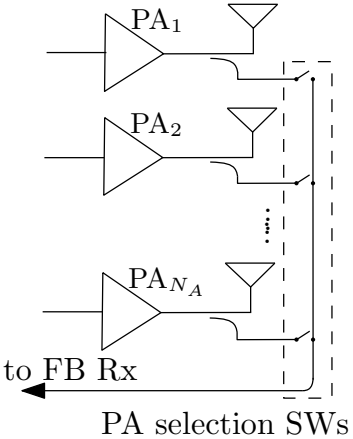

(d)

Fig. 2. Feedback strategies used for phased array DPD training that contains only a single digital chain (IQ). Architectures are listed as (a) OTA feedback, (b) underdetermined PA feedback, (c) RF-combined array feedback and (d) time-shared feedback.

\section{A. Over-the-air Feedback}

The principle of the OTA feedback is described in Fig. 2(a). The DPD trained with such a feedback is presented for example in [3], [8], [12], [13], [15], [16]. Use of reference antennas does not add post-PA losses from e.g., couplers and switches that would decrease the overall power efficiency. In order to get a proper estimate of the array behavior, the reference antenna should be placed in the far-field. A common definition for the far-field is that the phase error over the array aperture with respect to the observation in the farfield is less than $22.5^{\circ}$ [22]. For example, for antenna array of $\lambda / 2$-spaced ( $\lambda$ denotes the wavelength) antenna elements shaped to $16 \times 2$ formation, this means around 1.5 meters. On the other hand, depending on the FoM to be measured, the requirement can be relaxed without a significant impact on the performance [15], [31]. In case of array DPD, the error depends on the similarity of the nonlinear PA responses. Especially, if the responses are highly correlated, the phases of individual elements have smaller impact in combining, and therefore smaller measurement distance may be sufficient [15], [16].

The main drawback of using the reference antennas is the fact that the measurement is sensitive to multipath propagation and interference. The reference antenna is only placed in a certain steering angle, and the antenna may interfere with the actual beam formed by the array towards the end user. The feedback antenna or a probe can be also placed to the nearfield as in [13], where the authors use a coupling antenna surrounded by the actual Tx elements to sample individual PA outputs. From the RF implementation perspective, this offers a possibility to integrate the measurement $\mathrm{Rx}$ as a part of the actual array in a compact form factor. The drawback is that the coupling antenna can effectively only couple paths connected to the adjacent antenna elements without significant attenuation. However, low coupling from more distant antenna elements to the coupling antenna may be acceptable especially in high power systems. In principle, the usage of the coupling antennas can be considered to be an alternative implementation of the conventional directional couplers.

\section{B. Underdetermined PA Feedback}

Underdetermined, i.e. partial feedback means that the number of feedback paths is less than the number of the individual Tx paths. Such feedback approach is presented in Fig. 2(b). In some cases, even a single PA FB [10] can give decent estimate on the array behavior. In principle, if all the PAs have similar response and the antenna coupling is negligible, using a single PA FB gives the same response than the array feedback. However, in practice, there are always differences over the PAs which compromises the performance of the single PA FB in phased array transmitters as analyzed in [32]. Nevertheless, the number of feedback paths can also be more than one. For example, from the RF-layout perspective, the feedback paths can be allocated easily to the outermost elements of the array.

\section{Combined Array Feedback}

The ultimate feedback solution would be to emulate the channel in the feedback by recovering the channel phase and amplitude, and combining the paths to a single FB output as shown in Fig. 2(c). Such feedback schemes are proposed, for example, in [11] and [6]. In the latter one, the architecture is called anti-beamforming feedback, describing very well the functionality of the approach. The basic idea of the feedback is to cancel the phase shifts of the Tx paths, i.e. conjugate the beamformer. Conjugating the beamformer usually results in the original channel vector in case of the maximum ratio transmission (MRT) beamforming or a regular beam steering, traditionally used in the phased array systems. In principle, the feedback network can emulate different directions as well, enabling the conducted emulation of the beam formed by the array. The main drawback of the approach is that each feedback path should be equipped with an additional phase shifter, possible gain control element, and a symmetric combining network that eventually increases the complexity and may be rather challenging to implement in highly crowded RF layout. However, in TRx architectures that uses different paths for Tx and Rx, the system could eventually reuse the Rx paths for the feedback in transmit mode. 


\section{Time-shared Feedback}

Most of the OTA and combined feedback solutions aim at measuring all PA outputs simultaneously, i.e., they measure the actual radiated array response. Another approach is to use a simpler feedback solution and separate the measurements of the individual PA outputs in time. The switches shown in Fig. 2(d) can be used to couple each PA output to the feedback in different time instants. This has been proposed for example in [4], [5], and [7]. All state-of-the-art switchable or timeshared FB approaches use additional switches to connect the FB Rx to the PA outputs one-by-one. The main benefit is that this enables to have a model for each transmit path which may help to speed up the adaptation of the DPD for rapid changes in the steering angle. In addition, it is important to note that due to the asymmetry of the delays of the paths (phases) and gains, the feedback should be calibrated in order to map the measured response to the actual PA output and make the individual branches comparable.

\section{E. Common Feedback Line Utilizing TDD switches}

One further solution of the time-shared feedback is to reuse the TDD TRx architecture where the Tx and Rx paths are connected to the same antenna and separated by TRx switches. The principle is presented in Fig. 3. In this architecture, the feedback can be implemented as a single feedback line that couples each transmit path to the feedback Rx. Compared to the other time-shared feedback solutions, the architecture does not require any additional switches or other active electronics before the actual Rx (mixer, analog-to-digital (A/D) converters, etc.) which simplifies the implementation and control. This may be very attractive solution for phased array implementations, where the RF layout is extremely crowded. Hence, any additional component and control increase the system complexity, and compromise the layout solutions. The main drawback of this approach is that it is not able to characterize the PAs under varying load conditions caused by finite antenna coupling. However, for many cases, the architecture can be used for characterizing the individual PA paths and perform successful linearization. The principle of the approach is presented in detail in the following sections together with RF implementation and experimental validation.

\section{LOW-COUPLing Phased ARRAY TRANSMitTer With OTA-CALIBRATED COMMON FEEDBACK LINE}

\section{A. $28 \mathrm{GHz}$ Transceiver Module with GaN PAs}

A photograph of the 16-chain $28 \mathrm{GHz}$ phased array TRx PCB and 64-element antenna array is shown in Figs. 4 and 5, respectively [33]-[35]. Each of the 16 paths is connected to a $2 \times 2$-element fixed unit cell via sub-miniature push-on micro (SMPM) connectors. The unit cells have $\lambda$ spacing at $28 \mathrm{GHz}$. The isolation between the individual antenna ports is more than $35 \mathrm{~dB}$ [35]. In this paper, only half of the transmit paths are used and hence eight Tx paths drive 32 patch antenna elements. The used elements are surrounded by the white box in Fig. 5. The block chart of the array with eight TRx paths is shown in Fig. 6. The common transmit path of all paths consist

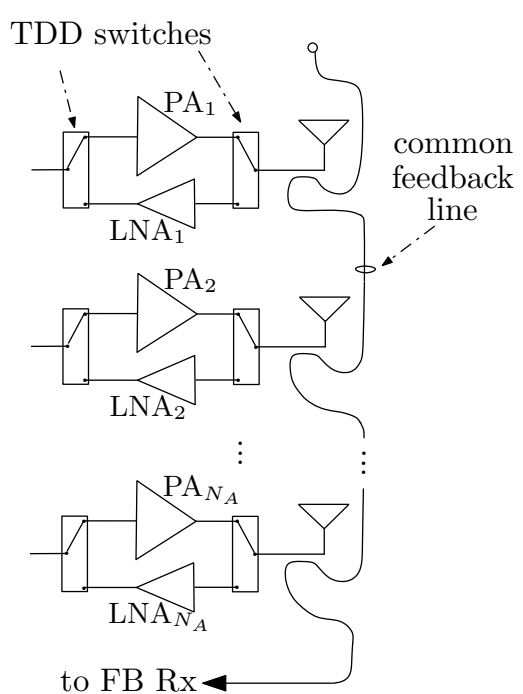

Fig. 3. Proposed feedback strategy for TDD systems. The individual PA measurements are separated in time by reusing the TDD switches of the array front end.

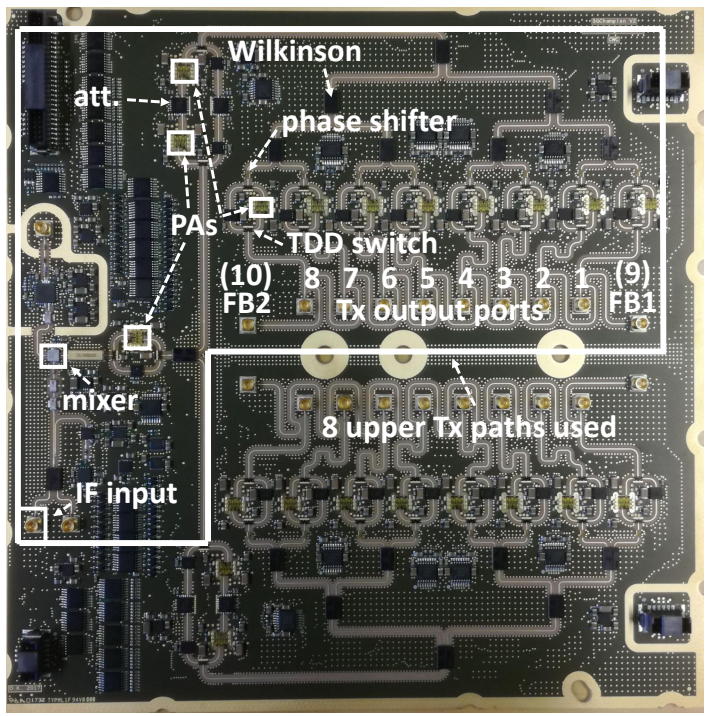

Fig. 4. Photograph of the $28 \mathrm{GHz}$ phased array TRx under test [21].

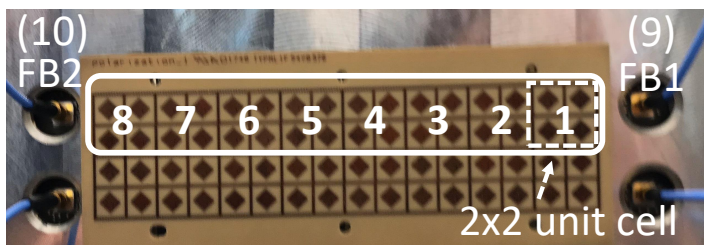

Fig. 5. 64-element antenna array [21]. The elements inside white box are used for transmission.

of HMC264LC3B mixer and TGA2595 Gallium Nitride (GaN) PA. After the PA, the signal is divided into two antenna rows by a PD-0530SM Wilkinson power divider. Both rows have two TGA2595 PAs separated by controllable 5-bit HMC939 attenuators to drive the Wilkinson power division network. The individual paths contain TGP2100 5-bit phase shifters and TGA2595 PAs. The Tx and Rx paths are separated by MASW011036 TRx switches such that Tx and Rx share the same 


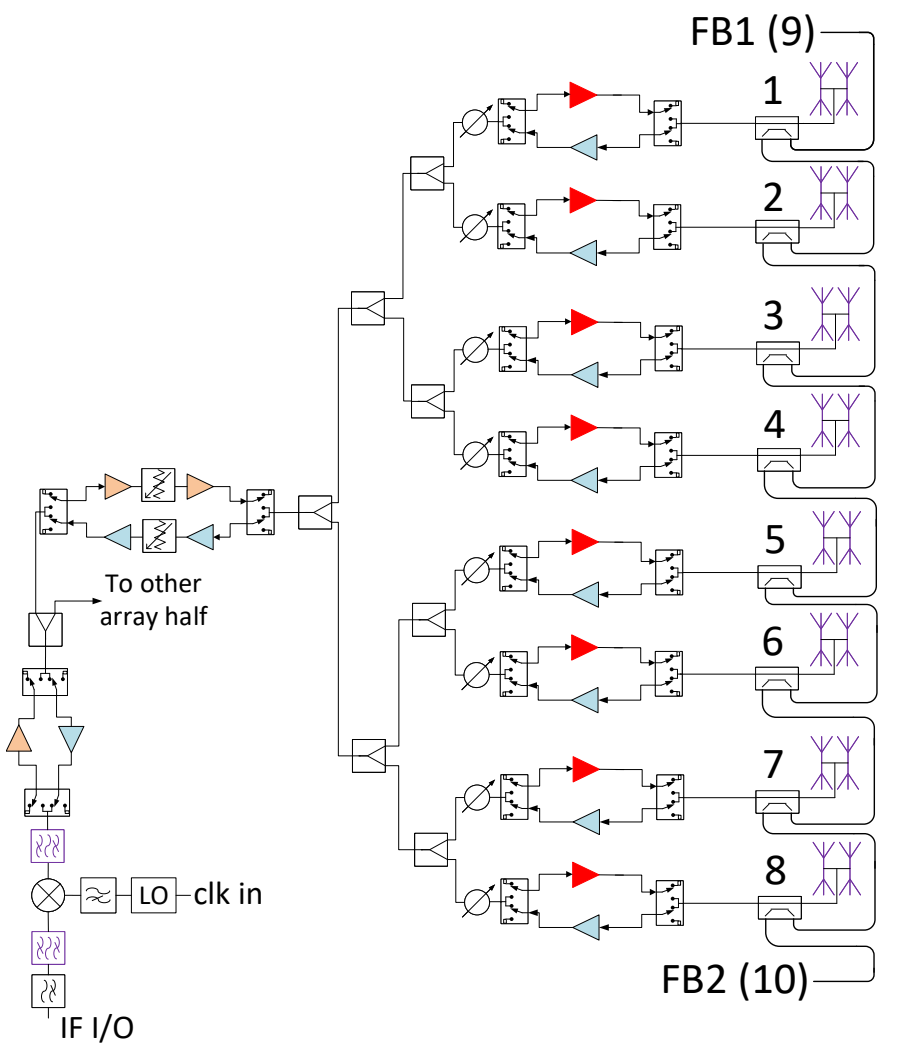

Fig. 6. Block chart of the $28 \mathrm{GHz}$ phased array TRx under test.

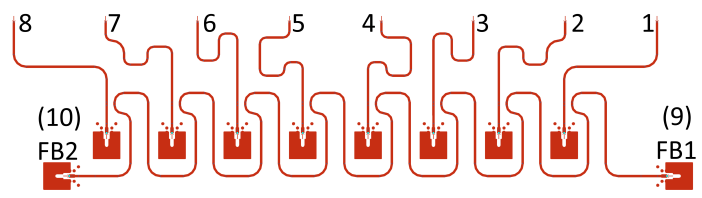

Fig. 7. Layout picture of the common FB line for eight transmit paths [21].

phase shifters, power division network and mixer. Meandering microstrip lines are used in the transmit paths to match the group delay from the common path to each antenna connector.

\section{B. Common Feedback Line}

The TRx shown in Fig. 4 contains two FB lines and one of them is used in this paper. Layout of the FB line is shown in Fig. 7. A meandering microstrip line is routed between SMPM antenna connectors and it couples to each PA output on the same side. Coupling is not directional as signals from individual PA output ports (1 to 8) are coupled to both FB line ports (FB1 and FB2). The coupling is designed to be as low as $40 \mathrm{~dB}$ such that the coupler itself does not increase the antenna coupling, and that it has minimum impact on the loss from PA output to the antenna input. Also, the coupling should be selected such that the noise does not dominate the signal ACPR when measured from the feedback path. The EM simulated (ADS Momentum) S-parameters from the TDD switch output to the feedback connectors FB1 and FB2 are presented in Figs. 8(a) and (b), respectively. Simulation ports 9 and 10 denote the feedback paths FB1 and FB2, respectively.

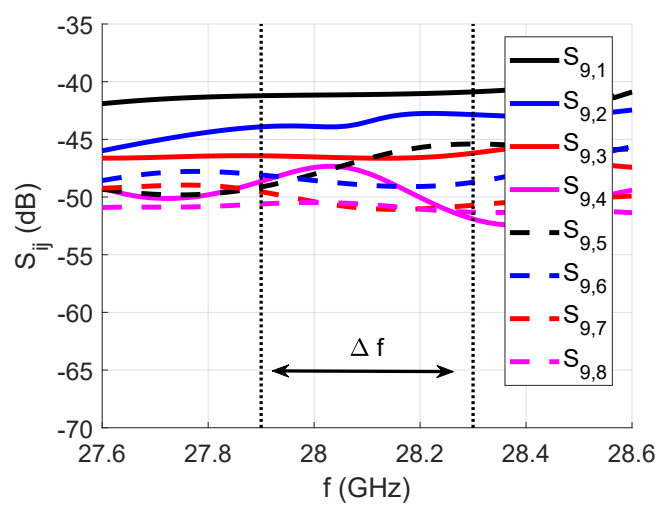

(a)

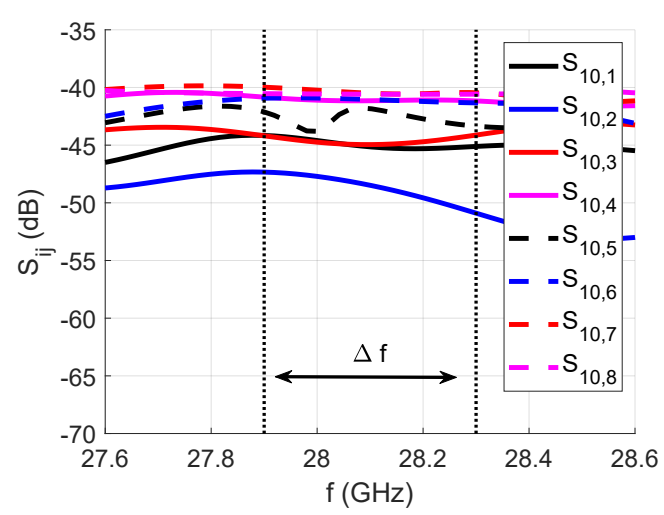

(b)

Fig. 8. EM-simulated feedback responses from different input ports for (a) feedback output FB1 and (b) FB2, respectively. Ports 9 and 10 are feedback outputs 1 and 2 .

The bandwidth of interest is $\Delta f=400 \mathrm{MHz}$ around $28.1 \mathrm{GHz}$ center frequency. The simulated loss from the nearest port to the FB output is around $40 \mathrm{~dB}$ for both feedback outputs whereas the loss from the farthest port is almost $50 \mathrm{~dB}$, as seen in Fig. 8. The simulated loss from the PA output port to the output connector is around $1 \mathrm{~dB}$ including the switch loss. For measuring individual transmit paths, FB1 is used to couple the signal transmitted by branches 1 to 4 and FB2 is used for the branches 5 to 8 , respectively. The selection is done simply based on the power measured at the feedback path. For the DPD training, it is important that all measurements have good and nearly similar signal-to-noise-ratio (SNR). This is due to the fact that the individual Tx outputs (including also the noise of each measurement) are later on weighted by the feedback calibration filters derived in section IV-C when training the DPD in section V.

\section{On-site Feedback Calibration by OTA-measurements}

The FB line used for DPD has gain and phase (delay) differences from the individual coupling points to the FB output. Hence, the coupled response from each PA is different. Moreover, the phase and amplitude of the feedback depend on the frequency which makes the behavior between the paths vary over the measured bandwidth. As the aim of the common DPD is to linearize the array in the far-field, the FB line should 


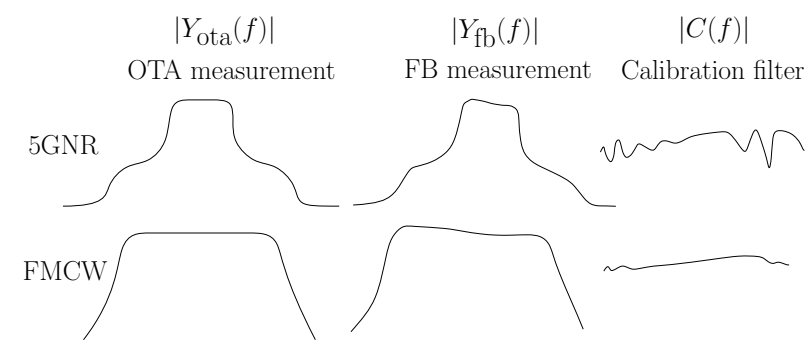

Fig. 9. Principles of frequency domain behavior of the feedback calibration filters when trained with FMCW and 5GNR signals against the feedback frequency response.

be calibrated by OTA measurements. The main emphasis is to compare the measurements performed through the feedback to the OTA measurements, and derive calibration filters to post-equalize the feedback measurements to mimic the OTA measured signals. The process is performed for each Tx path separately. Note that the behavior of the FB itself is static and linear. It depends only weakly on the steering angle due to the high antenna isolation and similar antenna elements. Hence, a single FB calibration is sufficient for multiple steering angles. However, DPD will be direction dependent taking into account the impacts of phase and amplitude control of each Tx path altogether.

1) Waveform Considerations for Feedback Calibration: In [21], the same 5GNR signal (100 MHz wide 256-QAM cyclic prefix (CP)-OFDM) was used for the FB calibration and DPD training. Hence, in [21] the selected training signal contains very little power on the adjacent channels. This means that when the feedback calibration coefficients were trained in [21], the calibration was not able to model the entire bandwidth of the feedback versus the OTA response. To overcome this, we use the FMCW signal to train the feedback calibration coefficients. Basically, the signal is a frequency sweep over the band of interest. The principles of the frequency domain behavior of the calibration filters with both 5GNR and FMCW signals are illustrated in Fig. 9. In the figure, $Y_{\text {ota }}(f)$ and $Y_{\mathrm{fb}}(f)$ represents the signal spectra when measured with reference antenna and the common feedback line, respectively. The calibration filter frequency response is derived as

$$
C(f)=\frac{Y_{\text {ota }}(f)}{Y_{\mathrm{fb}}(f)} .
$$

With 5GNR signal, the calibration filter response deviates significantly in the adjacent bands, which would cause further problems when the feedback measurements are post-equalized in DPD training. This has not been considered in [21] where only few taps were used in the calibration filter. On the contrary, with FMCW signal the filter frequency response remains relatively flat over the entire bandwidth as expected. The wider bandwidth also makes it easier to detect and remove potential multipath components that may be observed in the OTA measurements. For calibration of individual Tx paths, FMCW signal has been used for example in [36] and here similar principles are used for calibrating the feedback Rx.

2) OTA Measurement Setup for Feedback Calibration: A photograph of the measurement setup used for calibrating the

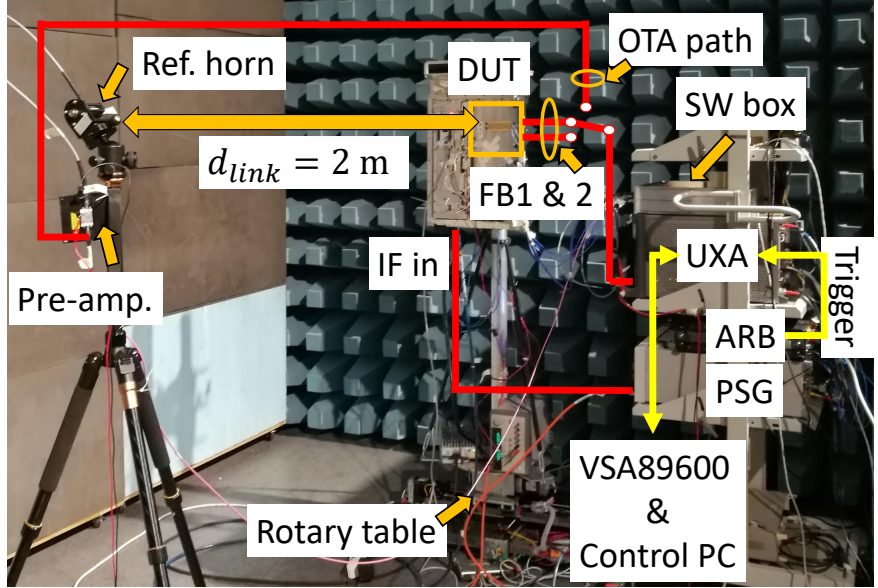

Fig. 10. Measurement setup for OTA calibration and DPD verification measurements.

feedback paths is presented in Fig. 10. The same measurement setup is later on used in the DPD verification measurements. A-Info LB-28-15 standard gain horn antenna placed at 2 meter distance from the array is used as reference Rx. The used distance satisfies the array far-field condition at the used center frequency of $28.1 \mathrm{GHz}$. The FMCW training signal is fed to the intermediate frequency (IF) input of the DUT by Keysight M8190A arbitrary waveform generator (ARB) and E8257B vector signal generator (PSG). The OTA Rx and common FB outputs are connected to the Keysight N9040B UXA signal analyzer via Keysight custom-built RF switch box that separates the feedback paths FB1 and FB2, and the OTA reference antenna path. The UXA signal analyzer is controlled by vector signal analyzer (VSA) software that enables to use the automatic gain control (AGC) of the VSA. AGC is used to maintain the SNR of the individual PA measurements with the feedback solution that has different loss from the individual Tx paths to the UXA input. Similar AGC arrangement could be designed for any feedback Rx taking bandwidth, coupler coupling, receiver nonlinearity, and noise into account. For the OTA path, CA2630-141 pre-amplifier was used to amplify the signal due to the high loss of the $\mathrm{mmW}$ cables. The oversampling ratio used in the $\mathrm{Rx}$ was four. The measurement automation is done in Matlab. The OTA Rx and the switch box are only used to calibrate the feedback line and verify the radiated DPD performance. After the calibration, the OTA Rx can be disconnected from the system.

3) Feedback Calibration Procedure: A flow chart of the calibration method is shown in Fig. 11. The calibration procedure transforms the FB output to the reference point in the far-field. We explain below the flow of the calibration measurements to train the finite impulse response (FIR) calibration filters. The TDD switches (Fig. 6) of the phased array are configured to enable each Tx path one-by-one while the other parallel PAs are terminated to $50 \mathrm{ohm}$. Each Tx path is measured from the FB output $\vec{y}_{\mathrm{fb}, 1}, \ldots, \vec{y}_{\mathrm{fb}, 8} \in \mathbb{C}^{1 \times N_{\mathrm{s}}}$, where $N_{\mathrm{s}}$ is the number of samples, and from the observation $\mathrm{Rx}$ $\vec{y}_{\text {ota }, 1}, \ldots, \vec{y}_{\text {ota }, 8} \in \mathbb{C}^{1 \times N_{\mathrm{s}}}$ placed to the far-field at the boresight direction. 


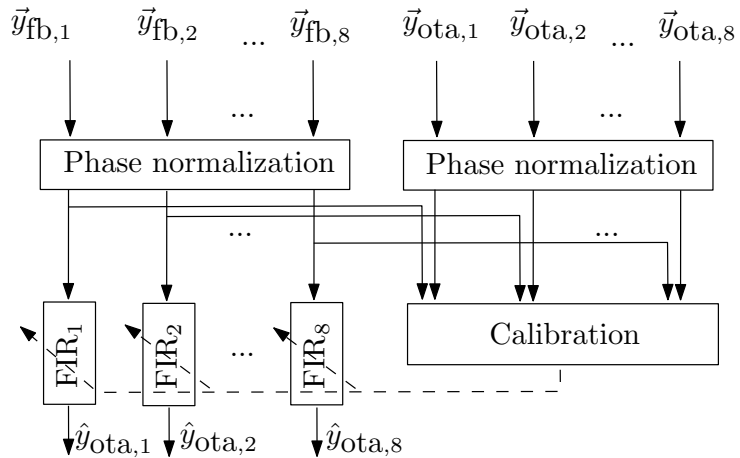

Fig. 11. Procedure for conducted FB OTA calibration.

In the calibration procedure, the measurements are first synchronized by using a simple cross-correlation method. However, the synchronization is only at one sample accuracy, and hence it is not able to recover the actual phase shift. Therefore, the phases of the FB signals are normalized against the common input signal $\vec{x}_{\text {in }} \in \mathbb{C}^{1 \times N_{\mathrm{s}}}$ as

$$
\vec{y}_{\mathrm{fb}, i, \text { norm }}=\vec{y}_{\mathrm{fb}, i} \exp \left(-j \phi_{\mathrm{cal}, i}\right),
$$

where

$$
\phi_{\mathrm{cal}, i}=\arg \left(\vec{x}_{\mathrm{in}}^{H} \vec{y}_{\mathrm{fb}, i}\right),
$$

where ()$^{H}$ stands for the Hermitean transpose and $\arg ()$ denotes the argument of a complex number. The phases of the OTA measured reference signals are normalized also by the same method. The purpose of the phase normalization is to model the coherent combining of the signals in the main lobe of the beam. The phase of the received waveform varies slightly due to the measurement setup as the used time domain triggering cannot make two measurements with different time instants to be phase coherent. This would require that at least two time-coherent receivers (a reference receiver and the feedback receiver).

The impulse responses (IRs) of the calibration filters are solved as

$$
\vec{c}_{i}=\mathcal{F}^{-1}\left(\frac{\mathcal{F}\left(\vec{y}_{\mathrm{ota}, i}\right)}{\mathcal{F}\left(\vec{y}_{\mathrm{fb}, i, \mathrm{norm}}\right)}\right),
$$

where $\mathcal{F}$ denotes the Fourier transform, $\mathcal{F}^{-1}$ is the inverse Fourier transform, and $\vec{y}_{\text {ota }, i}$ is the OTA measured signal from branch $i$. The discrete Fourier transforms of the digitally sampled signals were calculated via fast Fourier transform (FFT) in Matlab. After solving the calibration filters $\vec{c}_{i}$, the estimates of the modelled OTA FB signals are written as

$$
\hat{y}_{\mathrm{ota}, i}=\vec{c}_{i} * \vec{y}_{\mathrm{fb}, i, \mathrm{norm}},
$$

where $\hat{y}_{\text {ota }, i}$ denotes the estimate of the modelled OTA waveform of $i$ th Tx path and $(*)$ denotes the convolution. Hence, each FIR filter aims of converting the FB signal to the signal modelled in the array far-field in the main lobe direction. The FB post-equalization includes also the impact of the individual antenna elements.

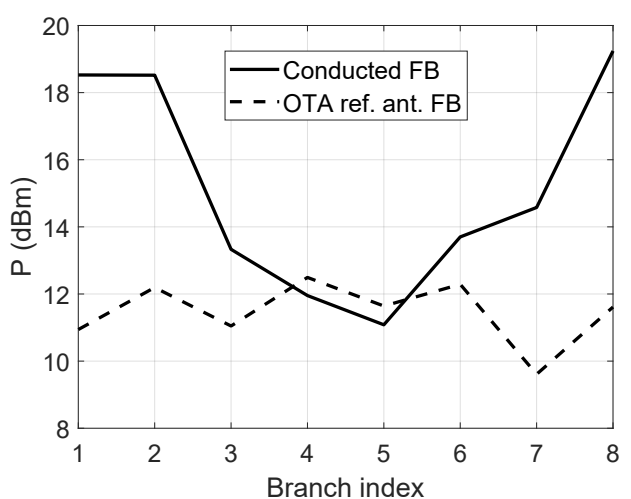

Fig. 12. EIRPs measured by OTA reference antenna and powers measured by the FB Rx. The gains of the reference antennas, pre-amplifier, and the loses of cables are compensated from the measurements to bring OTA and FB to same reference.

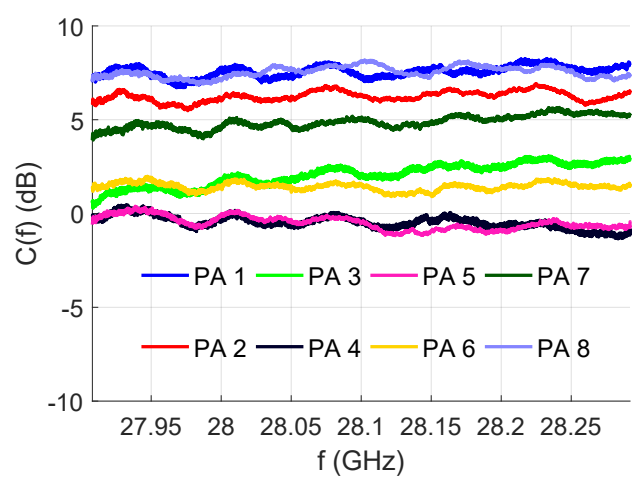

Fig. 13. Frequency responses of the calibration filters.

4) Feedback Calibration Measurements and Analysis: The OTA measured EIRPs and the powers measured from the feedback paths are plotted in Fig. 12. The reference antenna gain, preamplifier gain and the losses of the cables were compensated for to get the powers to the same reference. The measured OTA powers of the center antennas corresponded the power measured from the feedback path. Note that the calibration procedure can be performed even when the PAs are operating in their nonlinear regime. This is because the potential nonlinear impact is the same for the feedback and OTA measurements, when the PA branches are measured with a single element active only. Hence, the PA nonlinearities are normalized away in the calibration procedure and have no impact on the calibration filters. The frequency responses of the filters are seen in Fig. 13. Even though the filters are relatively flat in frequency, feedback paths 3 and 7 have slightly increasing slope with the frequency. Such behavior, if not compensated by the calibration, can cause additional post-PA memory to the DPD measurements, making it more difficult to linearize the system.

In principle, especially when performing the calibration onsite (i.e., on the place where the base station is installed), the calibration measurements may be affected by multipath propagation. For example, there may be reflections from the 


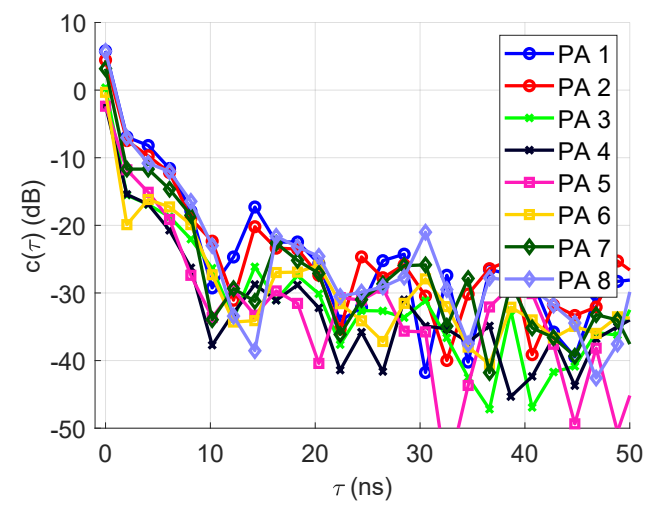

Fig. 14. Measured impulse responses of the calibration filters plotted over the first 25 taps.

environment, multi-bounce reflections between the reference antenna and the DUT etc. that should not be included to the calibration coefficients. This is important when the calibration filters are trained for individual antenna elements and hence the single-element transmit beams are not very directional. In order to verify the calibration environment and apply the calibration filters properly, the IRs of the calibration filters are analyzed. The IR data is plotted in Fig. 14. For clarity, only delays up to $50 \mathrm{~ns}$ (corresponds approximately 15 meters in free space) are shown as the rest are below the noise floor. With the used sampling rate this corresponds to 25 FIR taps (0.6 $\mathrm{m}$ per tap). In the calibration filters, only the FIR taps that are above the threshold of $25 \mathrm{~dB}$ below the main peak were considered to be relevant. In a multipath environment, one could use a delay-gate to remove the multipaths before calculating the calibration coefficients. If the feedback response is flat enough, taking only the strongest tap of the IR would be sufficient. Note that all paths are not necessarily physical as the inverse FFT (IFFT) has frequency/time leakage.

\section{Digital Predistortion of Phased Array with COMMOn FeEdBaCK Line}

\section{A. Memory Polynomial DPD Model}

As a DPD model, we use a memory polynomial (MP) with only odd-order nonlinear coefficients. The MP DPD output can be written as [37]

$$
x_{\text {in,dpd }}(n)=\sum_{m=0}^{M} \sum_{\substack{k=1 \\ k \text { :odd }}}^{K} d_{m, k} x(n-m)|x(n-m)|^{k-1},
$$

where $x_{\text {in,dpd }}(n) \in \mathbb{C}$ is the $n$th sample of the predistorted input, $x_{\text {in }}(n) \in \mathbb{C}$ denotes the $n$th sample of the raw input signal for the array, $K \in 2 \mathbb{Z}^{+}+1$ is the order of nonlinearity, $M \in \mathbb{Z}^{+}$is the memory depth, $n$ denotes the sample index, and $d_{m, k} \in \mathbb{C}$ denotes the $m$ th memory tap and $k$ th nonlinear order DPD coefficient. The DPD is trained by indirect learning architecture [37] by denoting

$$
\hat{x}_{M P}(n)=\sum_{m=0}^{M} \sum_{\substack{k=1 \\ k \text { :odd }}}^{K} d_{m, k} y(n-m)|y(n-m)|^{k-1},
$$

where $\hat{x}_{M P}(n)$ is the estimated input and $y(n)$ denotes the measured output signal. The DPD coefficients are solved by least squares (LS) fitting as follows. The observations $y(n)$ with different delays $m$ and nonlinear orders $k$ are stacked into a $M((K+1) / 2) \times N_{\mathrm{s}}$ matrix as

$$
\mathbf{Y}=\left(\begin{array}{cccc}
y_{1,0}(1) & y_{1,0}(2) & \ldots & y_{1,0}\left(N_{\mathrm{s}}\right) \\
y_{3,0}(1) & y_{3,0}(2) & \ldots & y_{3,0}\left(N_{\mathrm{s}}\right) \\
\vdots & \vdots & \ddots & \vdots \\
y_{K, 0}(1) & y_{K, 0}(2) & \ldots & y_{K, 0}\left(N_{\mathrm{s}}\right) \\
y_{1,1}(1) & y_{1,1}(2) & \ldots & y_{1,1}\left(N_{\mathrm{s}}\right) \\
\vdots & \vdots & \ddots & \vdots \\
y_{K, 1}(1) & y_{K, 1}(2) & \ldots & y_{K, 1}\left(N_{\mathrm{s}}\right) \\
\vdots & \vdots & \ddots & \vdots \\
y_{1, M}(1) & y_{1, M}(2) & \ldots & y_{1, M}\left(N_{\mathrm{s}}\right) \\
\vdots & \vdots & \ddots & \vdots \\
y_{K, M}(1) & y_{K, M}(2) & \ldots & y_{K, M}\left(N_{\mathrm{s}}\right)
\end{array}\right)^{T}
$$

where

$$
y_{k, m}(n)=y(n-m)|y(n-m)|^{k-1} .
$$

The overall DPD coefficients are solved as

$$
\hat{d}=\left(\mathbf{Y}^{H} \mathbf{Y}\right)^{-1} \mathbf{Y}^{H} \vec{x}_{\text {in }},
$$

where

$$
\begin{aligned}
\hat{d}= & {\left[d_{1,0}, d_{3,0}, \ldots, d_{K, 0},\right.} \\
& \left.d_{1,1}, \ldots, d_{K, 1}, \ldots, d_{, M}, \ldots, d_{K, M}\right]^{T},
\end{aligned}
$$

and $\vec{x}_{\text {in }}$ is the input vector.

\section{B. Three Alternative DPD Strategies Using the Common Feed- back Line}

When using the time-shared feedback approach, the array response has to be generated from the individual PA measurements taken in different time instants. Thus, there are several ways of generating the DPD object (14) from the measurements of the individual Tx paths. Three alternative strategies for generating the object and eventually the DPD coefficients for multiple transmit paths are presented in Fig. 15. The strategies are denoted as (i) directive array DPD, (ii) perPA DPD and (iii) stacked total radiated DPD. The strategies are explained in the following subsections. As a reference DPD, we use OTA trained DPD, where the complete array is trained with all elements active and the signal is measured by a reference antenna from the main lobe direction. The same MP model (13) is used for all cases. The measurements through the feedback are calibrated by the procedure presented in Fig. 11. The phase normalization described in (8) - (9) and calibration coefficients trained in the previous section are applied to the feedback outputs by (11).

1) Array DPD: Fig. 15(a) presents the directive array DPD strategy. The modelled array far-field response is used as the array output for the DPD training similarly as in [4]. The phase-normalized OTA estimates $\hat{y}_{\text {ota }, i}$ are simply summed to model the array response as

$$
\hat{y}_{\text {main lobe }}=\sum_{i=1}^{N_{\mathrm{A}}} \hat{y}_{\text {ota }, i},
$$




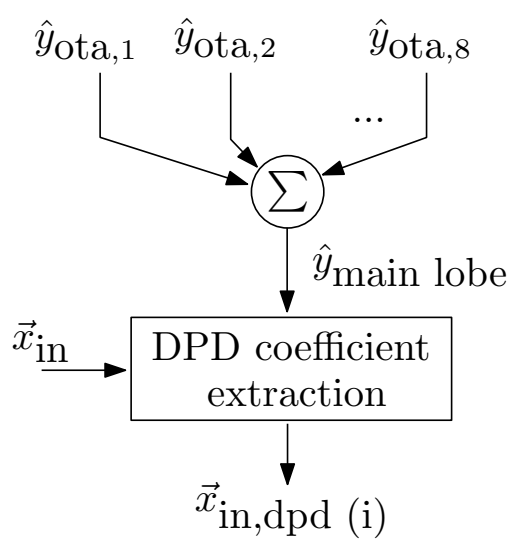

(a)

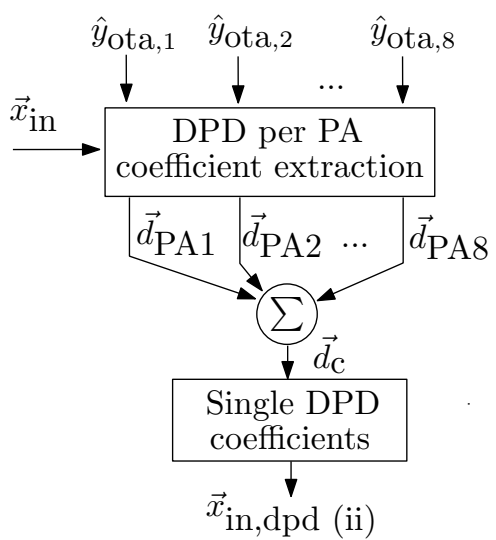

(b)

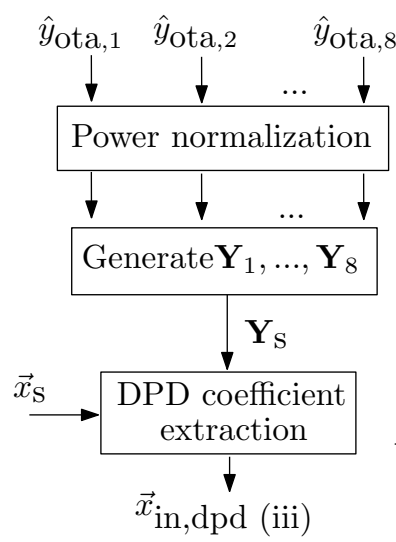

(c)

Fig. 15. Flow chart for generating the DPD object from individual PA measurements for (a) directive array DPD (method i), (b) Per-PA modelling based DPD (method ii), and (c) stacked total radiated DPD (method iii). In all cases the MP coefficients are solved by LS fitting.

and the DPD coefficients are solved with (16) by denoting $\vec{y}=\hat{y}_{\text {main lobe. }}$ In general, the approach is analogous to the methods that use combined array feedback [6], [11] or a reference antenna in the main lobe [9] to achieve single-input single-output (SISO) DPD. The main benefit of the approach is that only a single set of DPD coefficients are required for a static scenario. When applying the calibration filters derived in IV-C for the measurements of individual Tx paths, it is important that proper AGC is performed in the feedback Rx to ensure sufficient SNR for all measurements. This enables good SNR also for the array DPD when the outputs, and thus the noises, are combined.

2) DPD of Individual PAs: In the second strategy, the DPD coefficients are calculated for each PA, and the coefficient vectors are then combined to a single DPD polynomial. The approach is presented in Fig. 15(b). In the DPD calculation, all PA output powers are normalized by the same reference to model correctly the combining of slightly different levels of the PA outputs. The overall DPD model is calculated as

$$
\vec{d}_{(\mathrm{ii})}=\sum_{i=1}^{N_{\mathrm{A}}} \vec{d}_{\mathrm{PA} i},
$$

where $\vec{d}_{\mathrm{PA} i}$ denotes the DPD coefficients for Tx branch $i$. Method (ii) requires of calculating DPD coefficients for each PA and hence requires more mathematical operations than (i), despite of the actual DPD model used. On the other hand, modelling individual PAs may be beneficial for cases where the beamforming directions are rapidly changed as discussed in [5]. Note that compared to method (i), proper AGC is now even more important in the feedback Rx to maintain good SNR for the measurements of all individual Tx paths. This is especially true if very wide signal bandwidth is used.

3) DPD for Stacked PA Outputs: In DPD approach (iii), the responses of the individual Tx paths are treated as independent outputs. Hence, the aim is to linearize the total nonlinearity of the system. Basically, this means that the DPD coefficients are solved as LS solution over the PAs and time domain samples. The flow for generating the DPD object is shown in Fig. 15(c).
The outputs of the PAs are normalized and stacked to a matrix as

$$
\mathbf{Y}_{\mathrm{s}}=\left[\mathbf{Y}_{1}^{T}, \mathbf{Y}_{2}^{T}, \ldots, \mathbf{Y}_{N_{\mathrm{A}}}^{T}\right]^{T},
$$

where $\mathbf{Y}_{i}$ contains the output terms (14) of $i$ th PA. Similarly, the input signal $\vec{x}_{\text {in }}$ is repeated $N_{\mathrm{A}}$ times as

$$
\vec{x}_{s}=\left[\vec{x}_{\text {in }}^{T}, \vec{x}_{\text {in }}^{T}, \ldots, \vec{x}_{\text {in }}^{T}\right]^{T} .
$$

The overall DPD coefficients are solved by the LS fitting (16). Note that this is different than method used in [5] where the authors calculate LS solution over several azimuth angles in order to broaden the angular region of the linearization.

Obviously, in case of very different PA responses, the method (iii) compromises the main lobe linearity, but should achieve good total radiated nonlinearity. In addition, as method (iii) does not aim at directive linearization, it should be less sensitive to a varying steering angle. Method (iii) is similar to the approach proposed in [32]. However, the analysis in [32] contained only a rather limited set of mathematical simulations with simple memoryless PA models. Hence, we will verify the ability of the approach by OTA measurements in the following sections.

\section{OTA VERIFICATION OF DPD PERFORMANCE WITH BEAMSTEERING}

The setup used in the DPD verification measurement was the same as in the calibration measurements, and was shown in Fig. 10. The individual PA responses were measured via the feedback paths 1-2 and calibrated by the method presented in Section IV. The used input waveform was $100 \mathrm{MHz}$ wide 64-QAM CP-OFDM waveform following the 5G NR standard for FR2. The waveform has measured peak-to-average-powerratio (PAPR) of $10.9 \mathrm{~dB}$. In all experiments shown in this paper, the coefficients are trained over 48000 samples with four times oversampling (sampling rate $F_{s}=4 \cdot 1.2288$. $100 \mathrm{MHz}=491.52 \mathrm{MHz}$ ). The ACPR and EVM are calculated over a single sub-frame $(1 \mathrm{~ms})$ of the $5 \mathrm{G} \mathrm{NR}$ signal. The DUT is attached on a rotary table for beam measurements from $-45^{\circ}$ to $45^{\circ}$ with $1^{\circ}$ angular steps. When training the 
DPD, the phase shifters are set to steer the beam to $\phi_{s}$. The DUT was rotated to negative steering angle $-\phi_{s}$ for training the reference OTA DPD, but not for the DPD methods (i) - (iii) that use the feedback. The measurements were automatized by Matlab-based control software which controls the DUT, rotary table, and all measurement equipment. For beamforming measurements, the steering angles were swept from $-15^{\circ}$ to $15^{\circ}$ with $5^{\circ}$ steps.

\section{A. EIRP and EVM Performance}

Channel power beams with steering angles $-10^{\circ}, 0^{\circ}$, and $10^{\circ}$ are plotted in Figs. 16(a), (b) and (c), respectively. The corresponding EIRPs measured at the steering angles are shown in Table III. The impact of the DPD on the EIRP is negligible. The corresponding EVM beams are shown in Figs. 17(a), (b) and (c), respectively. Naturally, the EVM is high for the deep nulls while lower EVM is obtained in the main lobe and the sidelobes. All DPD methods are improving the EVM in the main lobe and the higher sidelobes. The numerical values of the main lobe EVM measured at the steering angles are shown in Table IV and plotted in Fig. 18. For successful transmission, 64-QAM requires better than $8 \%$ EVM [1]. All tested DPD methods improve the EVM over the specification threshold. OTA DPD trained to the steering angle gives naturally the best EVM in all measured cases. However, especially method (iii) provides EVM that is comparable to OTA DPD results. Modelling individual PAs (method (ii)) shows a slightly better performance than the array DPD (i). This is due to the fact that in (ii) the overall DPD model has eight different polynomials, whereas in (i) the complete array is modelled by a single polynomial.

\section{B. ACPR and Total Radiated Linearity}

The measured spectra in steering angles $-10^{\circ}, 0^{\circ}$ and $10^{\circ}$ are plotted in Figs. 19(a), (c) and (e). The corresponding main lobe ACPRs are shown in Table V. Method (iii) outperforms the other feedback methods in main lobe linearity in all tested scenarios. Similarly, as in the EVM measurements, using a polynomial to model each PA in method (ii) gives slightly better linearity in the steering angle than modelling the complete array by a single polynomial in (i).

The beams of the ACPR are plotted in Figs. 19(b), (d), and (f). The plotted $\mathrm{ACPR}_{\max }$ stands for the maximum of the lower and higher ACPR. With OTA DPD, ACPR is the best in the main lobe, indicating that the linearization is focusing to the steering angle. The same applies for method (iii). The best ACPR is usually achieved slightly away from the steering angle. However, the power in those directions is lower as was observed in Fig. 16. The peaks in the ACPR beams are in the nulls of the beam pattern. The methods (i) and (ii) do not have minima in the steering angles. This is due to the limited modelling accuracy and also due to some potential mutual impact between the PAs, as the DPD is trained by driving only one PA at a time. On the other hand, method (iii) performs well also as a function of the steering angle.

TRACPRs are calculated for each DPD scenario by integrating the power over the azimuth angles by using (5). The

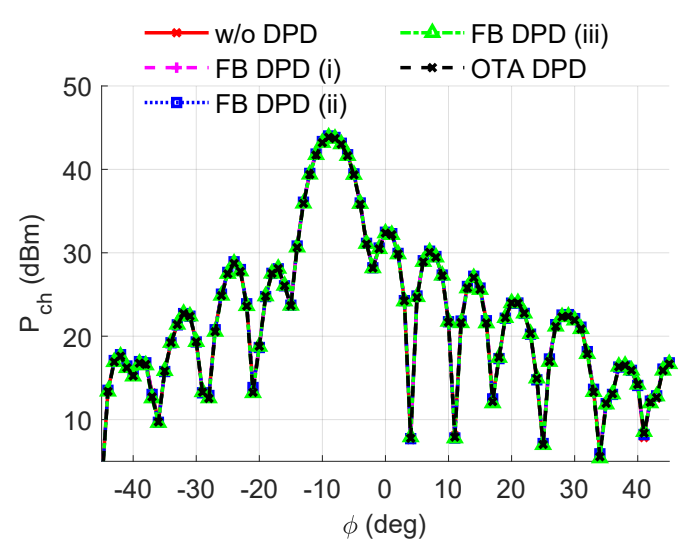

(a)

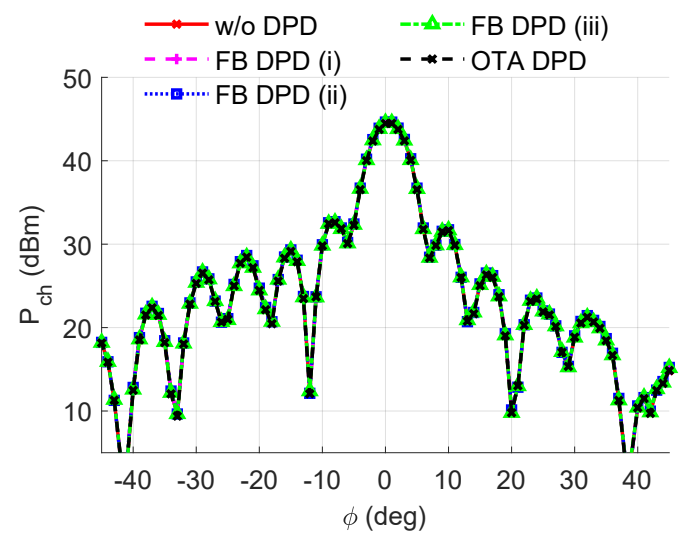

(b)

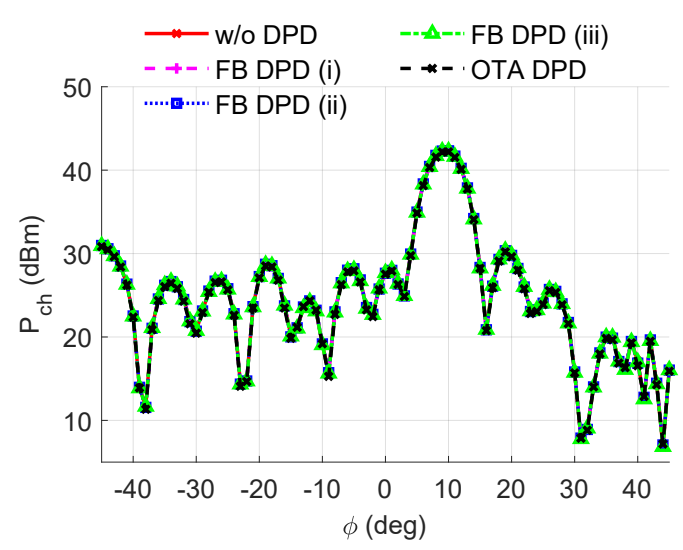

(c)

Fig. 16. Measured beam patterns of the $P_{\text {ch }}$ with steering angles (a) $\phi_{s}=$ $-10^{\circ}$, (b) $\phi_{s}=0^{\circ}$, and (c) $\phi_{s}=10^{\circ}$, respectively

TABLE III

EIRPS MEASURED IN THREE STEERING ANGLES WITH DIFFERENT DPD METHODS

\begin{tabular}{|c|c|c|c|}
\hline DPD method & $\phi_{s}=-10^{\circ}$ & $\phi_{s}=0^{\circ}$ & $\phi_{s}=10^{\circ}$ \\
\hline w/o DPD & $43.3 \mathrm{dBm}$ & $44.6 \mathrm{dBm}$ & $42.3 \mathrm{dBm}$ \\
\hline FB DPD (i) & $43.4 \mathrm{dBm}$ & $44.7 \mathrm{dBm}$ & $42.4 \mathrm{dBm}$ \\
\hline FB DPD (ii) & $43.3 \mathrm{dBm}$ & $44.6 \mathrm{dBm}$ & $42.3 \mathrm{dBm}$ \\
\hline FB DPD (iii) & $43.2 \mathrm{dBm}$ & $44.6 \mathrm{dBm}$ & $42.3 \mathrm{dBm}$ \\
\hline OTAdpd & $43.2 \mathrm{dBm}$ & $44.5 \mathrm{dBm}$ & $42.2 \mathrm{dBm}$ \\
\hline
\end{tabular}




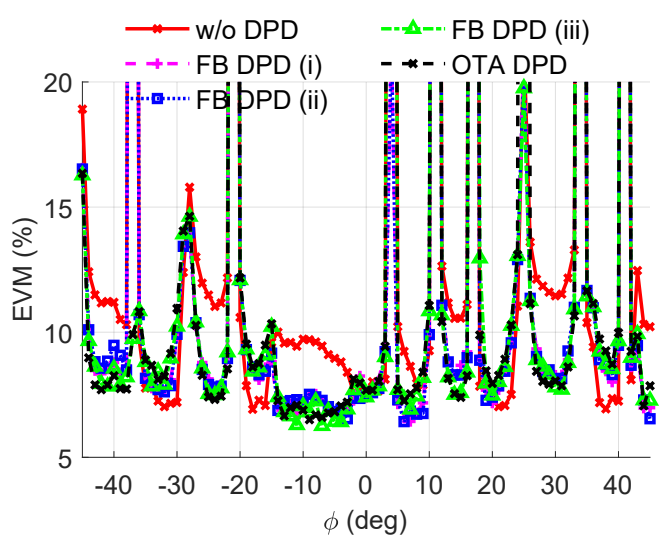

(a)

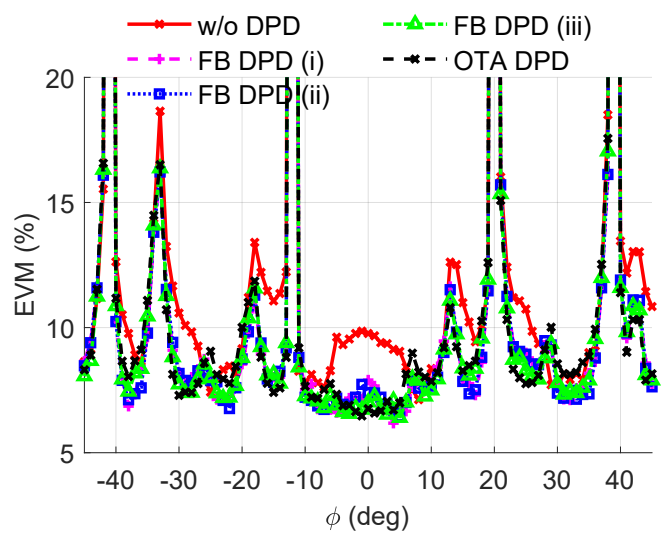

(b)

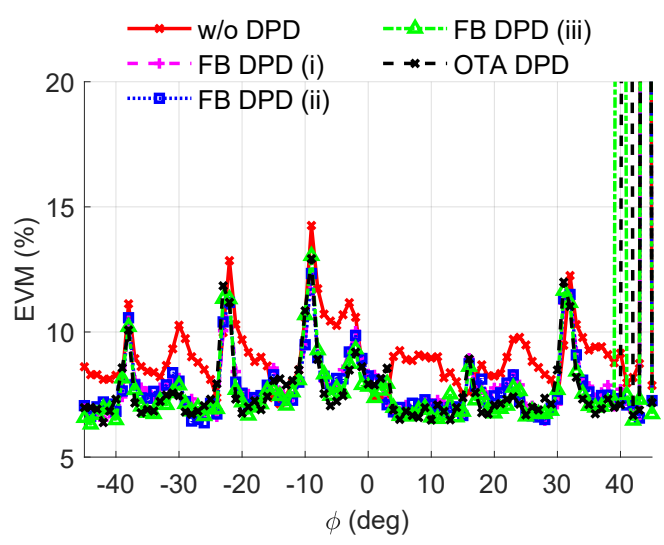

(c)

Fig. 17. Measured EVM at different spatial directions with steering angles (a) $\phi_{s}=-10^{\circ}$, (b) $\phi_{s}=0^{\circ}$, and (c) $\phi_{s}=10^{\circ}$, respectively

TABLE IV

MAIN LOBE EVMS MEASURED IN THREE STEERING ANGLES WITH DIFFERENT DPD METHODS.

\begin{tabular}{|c|c|c|c|}
\hline DPD method & $\phi_{s}=-10^{\circ}$ & $\phi_{s}=0^{\circ}$ & $\phi_{s}=10^{\circ}$ \\
\hline w/o DPD & $9.7 \%$ & $9.8 \%$ & $9.0 \%$ \\
\hline FB DPD (i) & $7.2 \%$ & $7.9 \%$ & $7.1 \%$ \\
\hline FB DPD (ii) & $7.2 \%$ & $7.4 \%$ & $7.1 \%$ \\
\hline FB DPD (iii) & $7.0 \%$ & $7.0 \%$ & $6.6 \%$ \\
\hline OTAdpd & $6.9 \%$ & $6.8 \%$ & $6.5 \%$ \\
\hline
\end{tabular}

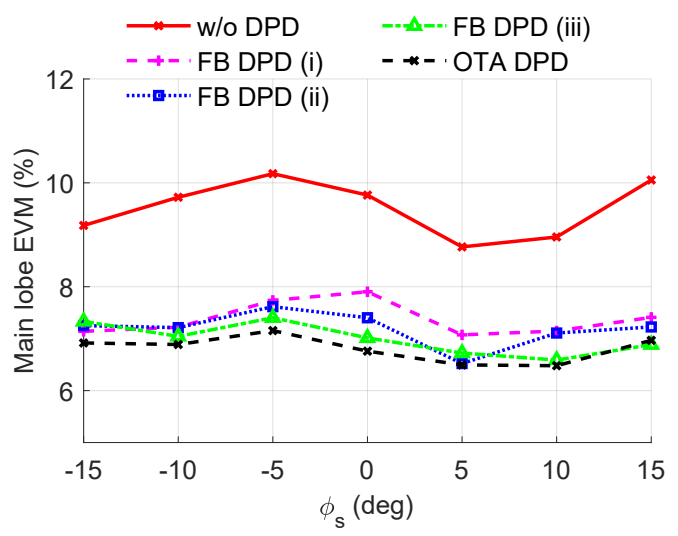

Fig. 18. EVM measured in the main lobe as a function of steering angle.

TABLE V

MEASURED MAIN LOBE ACPRS WITH THREE DIFFERENT STEERING ANGLES. SUB-INDICES L AND H STANDS FOR LOWER AND UPPER ADJACENT CHANNELS.

\begin{tabular}{|c|c|c|c|c|}
\hline DPD & FoM & $\phi_{s}=-10^{\circ}$ & $\phi_{s}=0^{\circ}$ & $\phi_{s}=10^{\circ}$ \\
\hline \multirow{2}{*}{ w/o DPD } & ACPR $_{\mathrm{L}}$ & $-29.7 \mathrm{~dB}$ & $-28.6 \mathrm{~dB}$ & $-31.2 \mathrm{~dB}$ \\
& ACPR $_{\mathrm{H}}$ & $-31.8 \mathrm{~dB}$ & $-32.6 \mathrm{~dB}$ & $-30.2 \mathrm{~dB}$ \\
\hline \multirow{2}{*}{ FB DPD (i) } & ACPR $_{\mathrm{L}}$ & $-36.2 \mathrm{~dB}$ & $-34.3 \mathrm{~dB}$ & $-37.4 \mathrm{~dB}$ \\
& ACPR $_{\mathrm{H}}$ & $-39.2 \mathrm{~dB}$ & $-39.1 \mathrm{~dB}$ & $-34.9 \mathrm{~dB}$ \\
\hline \multirow{2}{*}{ FB DPD (ii) } & ACPR $_{\mathrm{L}}$ & $-37.3 \mathrm{~dB}$ & $-34.4 \mathrm{~dB}$ & $-38.7 \mathrm{~dB}$ \\
& ACPR $_{\mathrm{H}}$ & $-39.8 \mathrm{~dB}$ & $-40.6 \mathrm{~dB}$ & $-37.0 \mathrm{~dB}$ \\
\hline \multirow{2}{*}{ FB DPD (iii) } & ACPR $_{\mathrm{L}}$ & $-41.1 \mathrm{~dB}$ & $-37.1 \mathrm{~dB}$ & $-39.9 \mathrm{~dB}$ \\
& ACPR $_{\mathrm{H}}$ & $-42.6 \mathrm{~dB}$ & $-40.8 \mathrm{~dB}$ & $-40.7 \mathrm{~dB}$ \\
\hline \multirow{2}{*}{ OTA DPD } & ACPR $_{\mathrm{L}}$ & $-40.1 \mathrm{~dB}$ & $-40.2 \mathrm{~dB}$ & $-42.5 \mathrm{~dB}$ \\
& ACPR $_{\mathrm{H}}$ & $-41.0 \mathrm{~dB}$ & $-38.2 \mathrm{~dB}$ & $-41.7 \mathrm{~dB}$ \\
\hline
\end{tabular}

power is calculated for both adjacent channels and the main channel individually. The values below the noise level, and the values outside the measurement range of $\phi \in\left[-45^{\circ}, 45^{\circ}\right]$ were set to 0 before the summing. The TRACPRs versus the steering angle are plotted in Fig. 20(a) and numerical results with three different steering angles are shown in Table VI. Without DPD, the TRACPR results and ACPRs measured at the steering angle are close to each other. However, with DPD, the TRACPR is generally less than the ACPR measured in the steering angle. Method (iii) outperforms methods (i) and (ii) also in terms of TRACPR. It should be noted that in some cases, method (iii) gives even better TRACPR than the OTA DPD trained at the steering angle. In many of the practical scenarios, focusing the linearization to the main lobe gives also good TRACPR. This is due to the fact that the main lobe has also the maximum absolute adjacent channel power. However, in case of significant differences over the PA branches, linearizing only the main lobe may compromise the ACPR performance as discussed in [11].

Approximation for the TRACPR was also calculated from the performance measurements through the feedback by switching the PAs on one-by-one and using (6) for estimating the total amount of distortion. The absolute powers of ACP and $P_{\mathrm{ch}}$ were calculated for the post-equalized outputs of the feedback. The estimated results are given in Fig. 20(b). When measured through the feedback, methods (i) and (ii) estimate the best TRACPR performance while method (iii) 


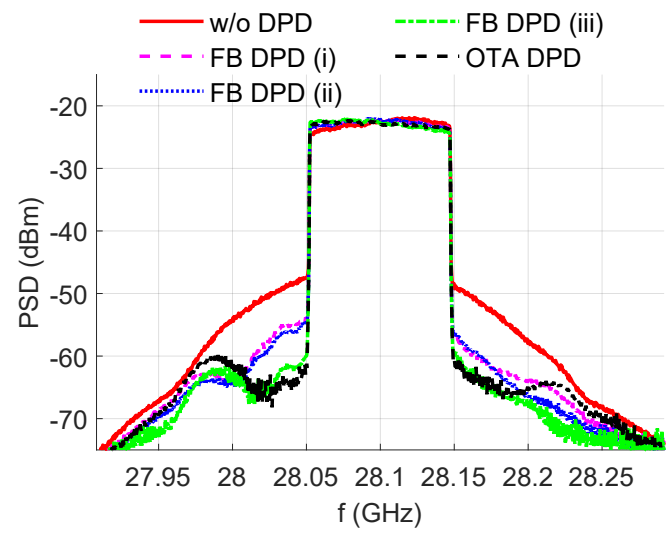

(a)

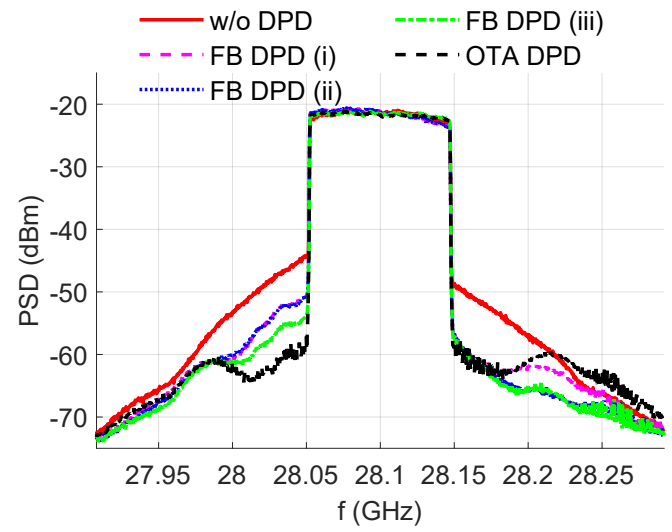

(c)

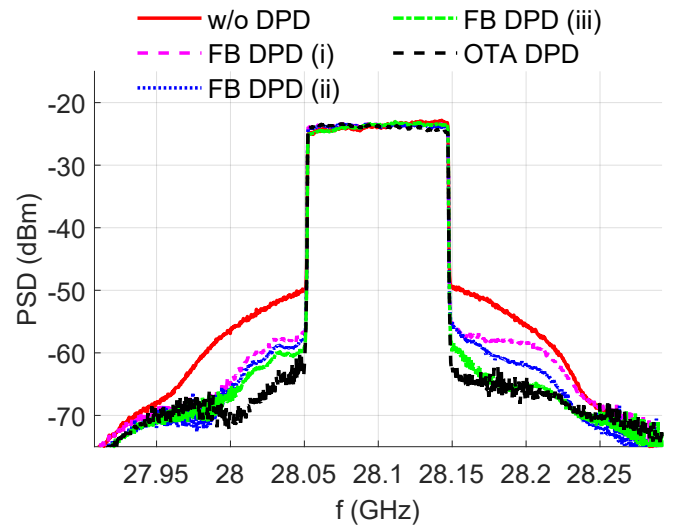

(e)

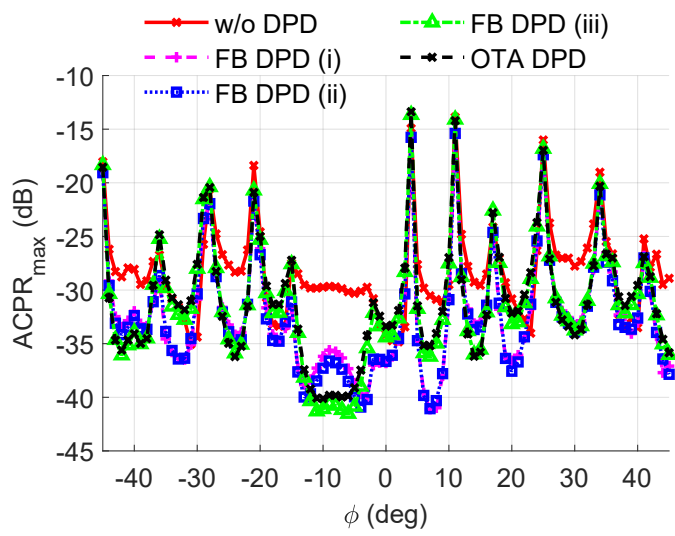

(b)

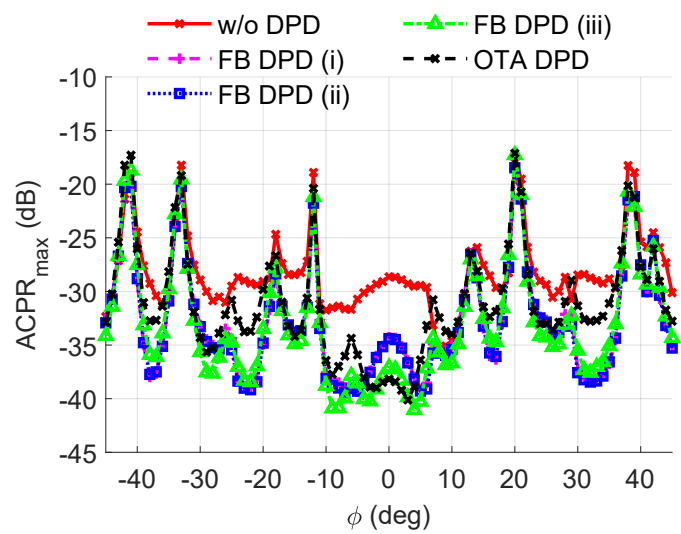

(d)

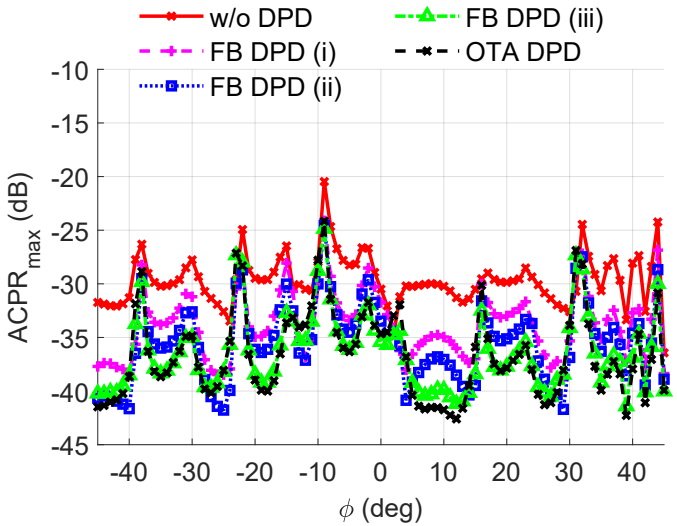

(f)

Fig. 19. Main lobe spectra and the measured beam patterns of ACPR with (a) - (b) $\phi_{s}=-10^{\circ}$, (c) - (d) $\phi_{s}=0^{\circ}$, and (e) - (f) $\phi_{s}=10^{\circ}$, respectively

and the OTA DPD estimate less linearization performance in individual PA outputs, when measured with only single element active at a time. Hence, feedback performance measurements overestimate the performance of the methods (i)(ii) due to the missing mutual impact of the PAs when all elements are active at the same time. The maximum difference between the measurement methods was around $4 \mathrm{~dB}$. In other words, the individual PA measurements should not be used for estimating the total DPD performance with very high accuracy, if the feedback approach is not able to measure the individual PAs with all elements active. However, it can be used to approximate the performance.

In addition to the TRACPR, also absolute CACPs, measured in terms of total power per frequency unit, were calculated from the results. The CACP is calculated as cumulative power over both $100 \mathrm{MHz}$ adjacent channels and divided by the bandwidth. The numeric values are shown in Table VI. The results are similar to TRACPR because DPD did not have 


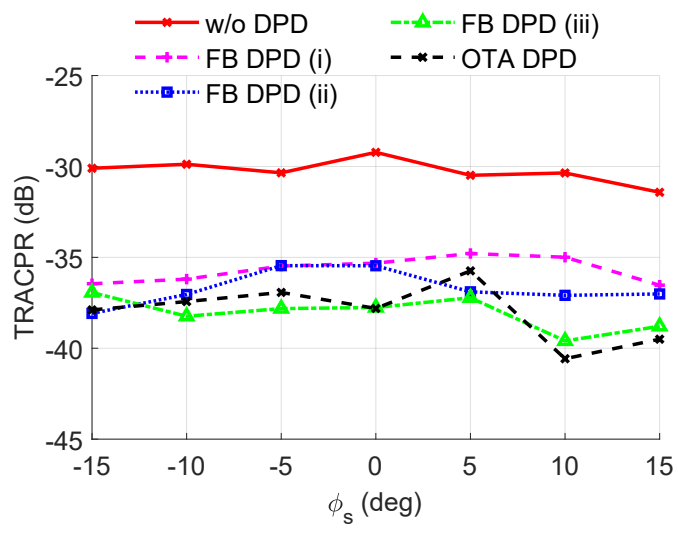

(a)

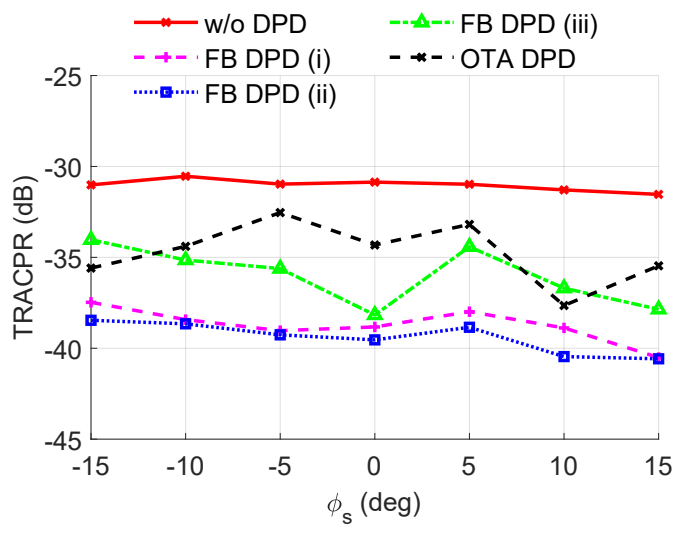

(b)

Fig. 20. (a) OTA measured TRACPR and (b) estimated TRACPR from the feedback. The used feedback approach over-estimates the DPD performances of methods (i) - (ii) while it underestimates the performance of method (iii) and OTA DPD.

TABLE VI

OTA-MEASURED TRACPRS AND CACPS WITH AND WITHOUT DPD. CACP IS GIVEN AS POWER PER MHZ

\begin{tabular}{|c|c|c|c|c|}
\hline DPD & FoM & $\phi_{s}=-10^{\circ}$ & $\phi_{s}=0^{\circ}$ & $\phi_{s}=10^{\circ}$ \\
\hline \multirow{2}{*}{ w/o DPD } & $\begin{array}{c}\text { TRACPR } \\
\text { CACP }\end{array}$ & $\begin{array}{c}-29.9 \mathrm{~dB} \\
-21.2 \mathrm{dBm}\end{array}$ & $\begin{array}{c}-29.2 \mathrm{~dB} \\
-21.6 \mathrm{dBm}\end{array}$ & $\begin{array}{c}-30.4 \mathrm{~dB} \\
-22.8 \mathrm{dBm}\end{array}$ \\
\hline \multirow{2}{*}{ FB DPD (i) } & $\begin{array}{c}\text { TRACPR } \\
\text { CACP }\end{array}$ & $\begin{array}{c}-36.2 \mathrm{~dB} \\
-27.5 \mathrm{dBm}\end{array}$ & $\begin{array}{c}-35.3 \mathrm{~dB} \\
-25.6 \mathrm{dBm}\end{array}$ & $\begin{array}{c}-35.0 \mathrm{~dB} \\
-27.4 \mathrm{dBm}\end{array}$ \\
\hline \multirow{2}{*}{ FB DPD (ii) } & $\begin{array}{c}\text { TRACPR } \\
\text { CACP }\end{array}$ & $\begin{array}{c}-37.0 \mathrm{~dB} \\
-28.3 \mathrm{dBm}\end{array}$ & $\begin{array}{c}-35.5 \mathrm{~dB} \\
-27.8 \mathrm{dBm}\end{array}$ & $\begin{array}{c}-37.1 \mathrm{~dB} \\
-29.5 \mathrm{dBm}\end{array}$ \\
\hline \multirow{2}{*}{ FB DPD (iii) } & $\begin{array}{c}\text { TRACPR } \\
\text { CACP }\end{array}$ & $\begin{array}{c}-38.2 \mathrm{~dB} \\
-29.7 \mathrm{dBm}\end{array}$ & $\begin{array}{c}-37.8 \mathrm{~dB} \\
-30.0 \mathrm{dBm}\end{array}$ & $\begin{array}{c}-39.6 \mathrm{~dB} \\
-32.0 \mathrm{dBm}\end{array}$ \\
\hline \multirow{2}{*}{ OTA DPD } & TRACPR & $-37.4 \mathrm{~dB}$ & $-37.8 \mathrm{~dB}$ & $-40.6 \mathrm{~dB}$ \\
& CACP & $-28.9 \mathrm{dBm}$ & $-30.3 \mathrm{dBm}$ & $-31.3 \mathrm{dBm}$ \\
\hline
\end{tabular}

significant impact on the overall TRP.

All measurement results indicate that simple conducted feedback architecture and training method where the PAs are measured individually, can provide 5G NR standard compatible linearization performance. Furthermore, the results are comparable to the DPD trained by using the reference antenna. The proposed approach offers fully passive feedback structure without using additional switches to separate the PA outputs, or combiners and phase shifters to sum them coherently at the feedback. Especially, the feedback approach as such is suitable for cases where the antenna isolation is high. In case of high antenna coupling, the active load impedance variations of individual PAs would not be included in the DPD training which could decrease the performance. For two-dimensional array, the system could partly overcome this by designing the feedback lines to meander between the Tx paths in vertical domain and separate the feedback lines in horizontal domain by RF switches. This would allow the system to measure the Tx paths with some of the adjacent elements active.

In practice, using the proposed feedback approach for DPD requires an additional training period for the DPD adaptation as only a single Tx path is active at a time. Hence, the DPD coefficients cannot be adapted during the normal operation of the Tx. Furthermore, the feedback strategy requires additional control for the TDD switches to enable only a single path at a time. The Tx paths can be trained in different training periods at different TDD slots, or within the same training period, depending on the DPD strategy and the available training time. The presented feedback calibration method applies also for calibration of other feedback receiver architectures if the Tx array itself enables switching individual paths on and off for the calibration period. This can be done in many ways, e.g. by switching elements on and off by TDD switches, decreasing the gains of the other Tx paths, or biasing other PAs down for the calibration period.

\section{CONClusion}

DPD requires a FB Rx to measure the nonlinear output of the Tx. For an array, the nonlinear output consists of multiple parallel elements. A common FB line architecture was proposed to be used for training the DPD by using the TDD switches of the array to enable measurements of individual array elements one-by-one. Compared to the existing FB architectures, the proposed approach does not need a large switch matrix to connect the coupled signal from each PA output to the FB. The individual Tx paths can be trained in different TDD time slots during a dedicated training period. The common FB line was calibrated by OTA measurements to transfer each measured FB signal to model the OTA measured main lobe signal.

Three different methods for generating the objective to linearize from the individual PA measurements through the feedback line were proposed. Method (i) combined the responses to model the main lobe, method (ii) calculated the DPD coefficients separately for individual PAs, and method (iii) stacked the individual PA outputs to calculate the DPD as the LS solution over the PAs. As a reference DPD, we used a DPD trained to the steering angle by using a reference antenna.

The performances of the DPD methods together with beamforming were verified by OTA measurements in terms of channel power, EVM and ACPR beams with different array steering angles. Furthermore, TRACPR and CACPR were calculated from the measurements by using a $100 \mathrm{MHz}$ wide 5G NR OFDM waveform. Linearization method (iii) 
outperformed the other methods trained through the feedback line, and its performance was comparable to the results with the OTA trained DPD. Compared to the reference antennas used to train the DPD, conductive feedback architectures are less sensitive to the interference in practical systems. The used feedback approach offered a low complexity RF layout as the common meandering microstrip was used to couple the PA outputs to the same feedback line. This may be an attractive solution in practice as it can be implemented without additional components with low cost and a small area.

\section{REFERENCES}

[1] 3GPP Tech. Spec. 38.104-2, "NR Base Station (BS) radio transmission and reception," v16.3.0 (Release 16), Mar. 2020.

[2] M. Latva-aho and K. Leppänen, Key drivers and research challenges for $6 G$ ubiquitous wireless intelligence. Oulu, Finland: University of Oulu, Sep. 2019.

[3] N. Tervo et al., "Digital predistortion concepts for linearization of mmw phased array transmitters," in Proc. 16th Int. Symp. Wireless Commun. Syst. (ISWCS), Aug. 2019, pp. 325-329.

[4] — "Digital predistortion of amplitude varying phased array utilising over-the-air combining," in IEEE MTT-S Int. Microw. Symp. (IMS) Dig., Jun. 2017, pp. 1165-1168.

[5] X. Liu et al., "Beam-oriented digital predistortion for 5G massive MIMO hybrid beamforming transmitters," IEEE Trans. Microw. Theory Tech., vol. 66, no. 7, pp. 3419-3432, Jul. 2018.

[6] M. Abdelaziz et al., "Digital predistortion for hybrid MIMO transmitters," IEEE J. Sel. Topics Signal Process., vol. 12, no. 3, pp. 445-454, Jun. 2018.

[7] S. Hesami et al., "Single digital predistortion technique for phased array linearization," in Proc. IEEE Symp. Circuits Syst. (ISCAS), May 2019, pp. 1-5.

[8] E. Ng, Y. Beltagy, P. Mitran, and S. Boumaiza, "Single-input singleoutput digital predistortion of power amplifier arrays in millimeter wave RF beamforming transmitters," in IEEE MTT-S Int. Microw. Symp. (IMS) Dig., Jun. 2018, pp. 481-484.

[9] E. Ng et al., "Digital predistortion of millimeter-wave RF beamforming arrays using low number of steering angle-dependent coefficient sets," IEEE Trans. Microw. Theory Tech., vol. 67, no. 11, pp. 4479-4492, Nov. 2019.

[10] L. Liu, W. Chen, L. Ma, and H. Sun, "Single-PA-feedback digital predistortion for beamforming MIMO transmitter," in Proc. IEEE Int. Conf. Microw. Millim. Wave Technol. (ICMMT), Jun. 2016, pp. 573-575.

[11] N. Tervo et al., "Analyzing the effects of PA variations on the performance of phased array digital predistortion," in Proc. IEEE 29th Annu. Int. Symp. Pers., Indoor Mobile Radio Commun. (PIMRC), Sep. 2018, pp. 215-219.

[12] E. Ng, A. B. Ayed, P. Mitran, and S. Boumaiza, "Single-input singleoutput digital predistortion of multi-user RF beamforming arrays," in IEEE MTT-S Int. Microw. Symp. (IMS) Dig., Jun. 2019, pp. 472-475.

[13] X. Liu et al., "Linearization for hybrid beamforming array utilizing embedded over-the-air diversity feedbacks," IEEE Trans. Microw. Theory Tech., pp. 1-14, Oct. 2019.

[14] W. Chen, G. Lv, X. Liu, and Z. Feng, "Energy-efficient doherty power amplifier MMIC and beamforming-oriented digital predistortion for 5G massive MIMO application," in Proc. Asia-Pacific Microw. Conf. (APMC), Jan. 2017, pp. 391-394.

[15] X. Wang, Y. Li, C. Yu, W. Hong, and A. Zhu, "Digital predistortion of 5G massive MIMO wireless transmitters based on indirect identification of power amplifier behavior with OTA tests," IEEE Trans. Microw. Theory Tech., vol. 68, no. 1, pp. 316-328, Nov. 2020.

[16] X. Wang et al., "Real-time single channel over-the-air data acquisition for digital predistortion of $5 \mathrm{G}$ massive MIMO wireless transmitters," in Proc. IEEE MTT-S Int. Wireless Symp. (IWS), May 2019, pp. 1-3.

[17] A. Brihuega et al., "Piecewise digital predistortion for mmwave active antenna arrays: Algorithms and measurements," IEEE Trans. Microw. Theory Tech., Jun. 2020, Early access.

[18] B. Sadhu et al., "A 28-GHz 32-element TRX phased-array IC with concurrent dual-polarized operation and orthogonal phase and gain control for 5G communications," IEEE J. Solid-State Circuits, vol. 52, no. 12 , pp. 3373-3391, Nov. 2017.
[19] S. Shahramian, M. J. Holyoak, A. Singh, and Y. Baeyens, "A fully integrated 384-element, 16-tile, W-band phased array with self-alignment and self-test," IEEE J. Solid-State Circuits, vol. 54, no. 9, pp. 24192434, Aug. 2019.

[20] T. Sowlati et al., "A 60-GHz 144-element phased-array transceiver for backhaul application," IEEE J. Solid-State Circuits, vol. 53, no. 12, pp. 3640-3659, Oct. 2018.

[21] N. Tervo et al., "Digital predistortion of millimeter-wave phased array transmitter with over-the-air calibrated simplified conductive feedback architecture," in IEEE MTT-S Int. Microw. Symp. (IMS) Dig., Jun. 2020, pp. $1165-1168$

[22] C. Balanis, Antenna Theory: Analysis and Design, 4th ed. Wiley, 2012.

[23] K. Hausmair, P. N. Landin, U. Gustavsson, C. Fager, and T. Eriksson, "Digital predistortion for multi-antenna transmitters affected by antenna crosstalk," IEEE Trans. Microw. Theory Tech., vol. 66, no. 3, pp. 15241535, Mar. 2018

[24] C. Fager et al., "Linearity and efficiency in 5G transmitters: New techniques for analyzing efficiency, linearity, and linearization in a 5G active antenna transmitter context," IEEE Microw. Mag., vol. 20, no. 5 , pp. 35-49, Apr. 2019.

[25] M. E. Leinonen, N. Tervo, M. Sonkki, and A. Pärssinen, "Quality analysis of antenna reflection coefficient in massive MIMO antenna array module," in Proc. Eur. Microw. Conf., Sep. 2018, pp. 1553-1556.

[26] T. Tuovinen, N. Tervo, and A. Pärssinen, "Analyzing 5G RF system performance and relation to link budget for directive MIMO," IEEE Trans. Antennas Propag., vol. 65, no. 12, pp. 6636-6645, Dec. 2017.

[27] M. E. Leinonen, M. Jokinen, N. Tervo, O. Kursu, and A. Pärssinen, "System EVM characterization and coverage area estimation of 5G directive mmw links," IEEE Trans. Microw. Theory Tech., vol. 67, no. 12, pp. 5282-5295, Dec. 2019.

[28] 3GPP Tech. Spec. 38.903, "NR; Derivation of test tolerances and measurement uncertainty for User Equipment (UE) conformance test cases," v16.3.0 (Release 16), Mar. 2020.

[29] 3GPP Tech. Spec. 38.141-2, "NR; Base Station (BS) conformance testing Part 2: Radiated conformance testing," v16.3.0 (Release 16), Mar. 2020.

[30] B. Xu et al., "Analysis of impacts of expected RF EMF exposure restrictions on peak EIRP of $5 \mathrm{G}$ user equipment at $28 \mathrm{GHz}$ and 39 GHz bands," IEEE Access, vol. 7, pp. 20 996-21 005, Feb. 2019.

[31] H. Kong, Z. Wen, Y. Jing, and M. Yau, "Midfield over-the-air test: A new OTA RF performance test method for 5G massive MIMO devices," IEEE Trans. Microw. Theory Tech., vol. 67, no. 7, pp. 2873-2883, May 2019.

[32] S. Lee et al., "Digital predistortion for power amplifiers in hybrid MIMO systems with antenna subarrays," in Proc. IEEE 81st Veh. Technol. Conf. (VTC Spring), May 2015, pp. 1-5.

[33] M. E. Leinonen et al., " $28 \mathrm{GHz}$ wireless backhaul transceiver characterization and radio link budget," ETRI J., vol. 40, no. 15, pp. 89-100, Feb. 2018.

[34] O. Kursu et al., "Design and measurement of a 5G mmw mobile backhaul transceiver at $28 \mathrm{GHz}$," EURASIP J. Wireless Commun. Netw. vol. 2018, no. 1, p. 201, Aug. 2018.

[35] M. Sonkki et al., "Linearly polarized 64-element antenna array for mmwave mobile backhaul application," in Proc. Eur. Conf. Antennas Prop. (EUCAP), Apr. 2018, pp. 1-5.

[36] P. Ramabadran, S. Madhuwantha, P. Afanasyev, R. Farrell, L. Marco, S. Pires, and J. Dooley, "Digitally assisted wideband compensation of parallel RF signal paths in a transmitter," in Proc. ARFTG Microw. Meas. Conf., Jun. 2018, pp. 1-4.

[37] X. Huang, Z. Zhu, and H. Leung, Signal Processing for RF Circuit Impairment Mitigation, ser. Artech House mobile communications. Artech House Publishers, 2014. 


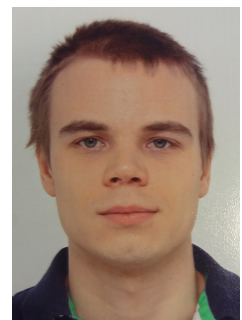

Nuutti Tervo (S'16) received the B.Sc. (Tech.) and M.Sc. (Tech.) degrees in electrical engineering from the University of Oulu in 2014, where he is currently pursuing the $\mathrm{PhD}$ degree with the Centre for Wireless Communications (CWC) research unit. He has a strong background in different fields of wireless communications including $\mathrm{RF}$, radio channel modeling, signal processing, and system-level analysis. Around those topics, he has already authored or co-authored more than 35 international journal and conference papers. His $\mathrm{Ph} . \mathrm{D}$ dissertation research focuses on nonlinearity and linearization of millimeter-wave beamforming transceivers.

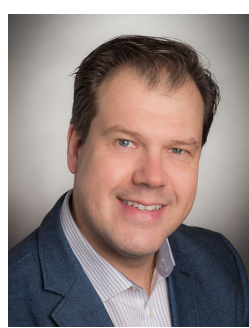

Marko E. Leinonen received the M.Sc. and Licentiate in Technology degrees in electrical engineering from the University of Oulu, Finland, in 1996 and 2002. From 1994 to 2012 he was with Nokia Mobile Phones, Oulu, Finland working with various positions with radio engineering and technology management. From 2006 to 2007, he was a Senior Engineering Manager in Bangalore, India. From 2012 to 2016 he was a Master Developer with Ericsson, Oulu, Finland. Since 2017, he has been with University of Oulu, Centre for Wireless Communications, Oulu, Finland where he is currently a research manager. His research interests include wireless radio systems and quality topics in radio engineering. He holds 40 granted international patent families concentrating on radio engineering.

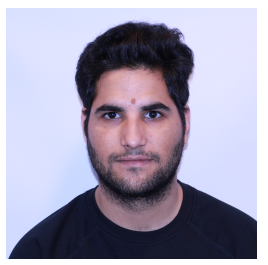

Bilal Khan received the B.Sc degree in Electrical Engineering (Communication) from University of Engineering and Technology Peshawar Pakistan in 2013, and the M.Sc. degree in Wireless Communication Engineering from University of Oulu, Finland, in 2016. He is currently pursuing the Ph.D. degree at the University of Oulu. His research interest includes digital predistortion for RF power amplifiers with emphasis on robust and simplified linearization solutions for multi-antenna transmitters in millimeterwave systems.

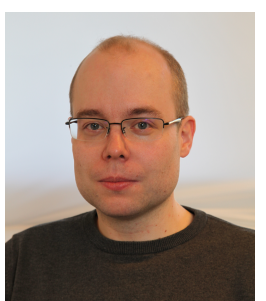

Olli Kursu received his MSc and $\mathrm{DrSc}$ degrees in electrical and electronics engineering from the University of Oulu, Finland, in 2006 and 2015, respectively. Currently, he is working as a postdoctoral researcher at the Centre for Wireless Communications, University of Oulu. His research interests include $\mathrm{mmW}, \mathrm{RF}$, analog, and mixed signal circuit design for wireless communication systems.

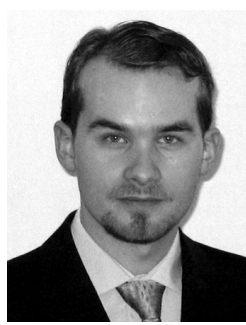

Janne P. Aikio Janne P. Aikio was born in Haukipudas, Finland, in 1977. He received the M.Sc. and $\mathrm{Ph} . \mathrm{D}$. degrees in electrical engineering from the University of Oulu, Oulu, Finland, in 2002 and 2007, respectively. He is Currently a Postdoctoral Researcher with the University of Oulu. His technical interests lie in the field of millimeter-wave integrated power amplifiers and modeling and analysis of distortion of RF power amplifiers.

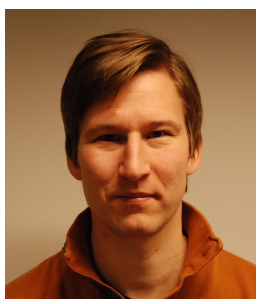

Markku Jokinen graduated as M.Sc. in Electronics from the University of Oulu in 2010 and he is working as a Research Scientist at Centre for Wireless Communications. He is pursuing the Doctoral degree and his has been recently focusing on the measurement techniques. He has also gained experience on the design and implementation of wireless system algorithms and protocols with software-defined radio platforms.

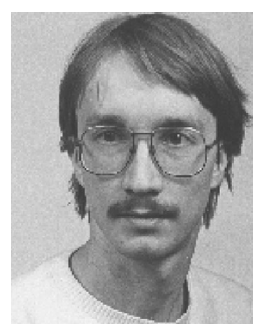

Timo Rahkonen was born in Jyvaskylä, Finland, 1962. He received his Diploma Engineer, Licentiate and Doctor of Technology degrees in 1986, 1991 and 1994, respectively, in University of Oulu, Finland. Currently he is a professor in circuit theory and circuit design in University of Oulu, Oulu, Finland, conducting research on linearization and error correction techniques for RF power amplifiers and $\mathrm{A} / \mathrm{D}$ and D/A converters

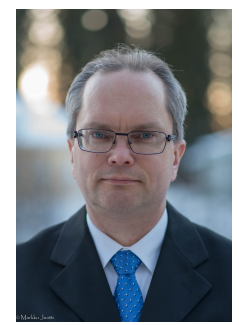

Markku Juntti (S'93-M'98-SM'04-F'20) received his M.Sc. (EE) and Dr.Sc. (EE) degrees from University of Oulu, Oulu, Finland in 1993 and 1997, respectively.

Dr. Juntti was with University of Oulu in 1992-98. In academic year 1994-95, he was a Visiting Scholar at Rice University, Houston, Texas. In 1999-2000, he was a Senior Specialist with Nokia Networks. Dr. Juntti has been a professor of communications engineering since 2000 at University of Oulu, Centre for Wireless Communications (CWC), where he leads the Communications Signal Processing (CSP) Research Group. He also serves as Head of CWC - Radio Technologies (RT) Research Unit. His research interests include signal processing for wireless networks as well as communication and information theory. $\mathrm{He}$ is an author or co-author in almost 500 papers published in international journals and conference records as well as in books Wideband CDMA for UMTS in 2000-2010, Handbook of Signal Processing Systems in 2013 and 2018 and 5G Wireless Technologies in 2017. Dr. Juntti is also an Adjunct Professor at Department of Electrical and Computer Engineering, Rice University, Houston, Texas, USA.

Dr. Juntti was an Editor of IEEE TRANSACTIONS ON COMMUNICATIONS and an Associate Editor for IEEE TRANSACTIONS ON VEHICULAR TeChNOLOGY. He was Secretary of IEEE Communication Society Finland Chapter in 1996-97 and the Chairman for years 2000-01. He has been Secretary of the Technical Program Committee (TPC) of the 2001 IEEE International Conference on Communications (ICC), and the Chair or CoChair of the Technical Program Committee of several conferences including 2006 and 2021 IEEE International Symposium on Personal, Indoor and Mobile Radio Communications (PIMRC), the Signal Processing for Communications Symposium of IEEE Globecom 2014, Symposium on Transceivers and Signal Processing for 5G Wireless and mm-Wave Systems of IEEE GlobalSIP 2016, ACM NanoCom 2018, and 2019 International Symposium on Wireless Communication Systems (ISWCS). He has also served as the General Chair of 2011 IEEE Communication Theory Workshop (CTW 2011). 


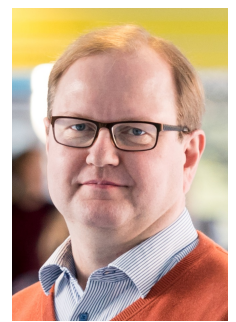

Aarno Pärssinen (S'95-M'01-SM'11) received the M.Sc., Licentiate in Technology and Doctor of Science degrees in electrical engineering from the Helsinki University of Technology, Finland, in 1995, 1997 , and 2000, respectively.

From 1994 to 2000 he was with Electronic Circuit Design Laboratory, Helsinki University of Technology, Finland, working on direct conversion receivers and subsampling mixers for wireless communications. In 1996, he was a Research Visitor at the University of California at Santa Barbara. From 2000 to 2011 he was with Nokia Research Center, Helsinki, Finland. During 2009-2011 he served as a member of Nokia CEO Technology Council. From 2011 to 2013, he was at Renesas Mobile Corporation, Helsinki, Finland working as a Distinguished Researcher and RF Research Manager. From October 2013 to September 2014 he was an Associate Technical Director at Broadcom, Helsinki, Finland. Since September 2014 he has been with University of Oulu, Centre for Wireless Communications, Oulu, Finland where he is currently a Professor. He is currently leading Devices and Circuits research area in 6G flagship program financed by Academy of Finland. His research interests include wireless systems and transceiver architectures for wireless communications with special emphasis on the RF and analog integrated circuit and system design.

Aarno Pärssinen has authored and co-authored one book, two book chapters, more than 150 international journal and conference papers and holds several patents. He is also one of the original contributors to Bluetooth low energy extension, now called as BT LE. He served as a member of the technical program committee of Int. Solid-State Circuits Conference in 2007-2017, where he was the chair of European regional committee in 2012-13, and the chair of the wireless sub-committee in 2014-2017. He has been serving as Solid-State Circuits Society representative for IEEE 5G initiative in 20152019. 